\title{
Anomaly detection on Activities of Daily Living with Linear Drift
}

\author{
Óscar Belmonte-Fernández • Antonio \\ Caballer-Miedes • Eris Chinellato . \\ Raúl Montoliu • Emilio Sansano-Sansano . \\ Rubén García-Vidal
}

Received: date / Accepted: date

\author{
Abstract \\ Background: Anomaly detection in Activities of Daily Living (ADL) plays \\ an important role in e-health applications. An abrupt change in ADL taken by \\ a subject might indicate that she/he needs some help. Another important issue \\ related with e-health application is the case where the change in ADL experiments \\ a linear drift, this is the case in cognitive decline, Alzheimer disease or dementia. \\ Methods: This work presents a novel method for detecting a linear drift in \\ ADL modeled as circular normal distributions. The method is based on techniques \\ commonly used in Statistical Process Control, and is able to detect and estimate \\ the change point in time when a linear drift started.

\footnotetext{
Ó. Belmonte-Fernández

Institute of New Imaging Technologies and Department of Computer Languages and Systems, Jaume I University, Castellón de la Plana, 12071, Spain

Tel.: +34-964-728315

Fax: +34 964728435

E-mail: oscar.belmonte@uji.es

A. Caballer-Miedes

Institute of New Imaging Technologies and Department of Developmental, Educational and Social Psychology and Methodology, Jaume I University, Castellón de la Plana, 12071, Spain E-mail: caballer@uji.es

E. Chinellato

Department of Design Engineering \& Maths, Middelsex University, London NW4 4BT, UK

E-mail: E.Chinellato@mdx.ac.uk

R. Montoliu

Institute of New Imaging Technologies and Department of Computer Architecture and Technology, Jaume I University, Castellón de la Plana, 12071, Spain

E-mail: montoliu@uji.es

E. Sansano-Sansano

Institute of New Imaging Technologies, Jaume I University, Castellón de la Plana, 12071, Spain E-mail: esansano@uji.es

R. García-Vidal

Institute of New Imaging Technologies, Jaume I University, Castellón de la Plana, 12071, Spain E-mail: vidalr@uji.es
} 
Results: Public data sets have been used to asses that ADLs can be modeled by a mixture of circular normal distributions. Exhaustive experimentation were performed on simulated data to asses the validity of the change detection algorithm, showing that the difference between the real change point and the estimated change point was $4.90_{+3.17}^{-1.98}$ days on average.

Conclusion: ADLs might be modeled using a mixture of circular normal distributions. A new method to detect anomalies following a linear drift is presented. Exhaustive experiments showed that this method is able to estimate the change point in time for processes following a linear drift.

Keywords Anomaly detection - Activities of Daily Living (ADL) - Abrupt change $\cdot$ Linear drift $\cdot$ Circular normal distribution.

\section{Introduction}

[1]

Anomaly detection in ADL is a particularly important issue in e-health applications such as elderly monitoring [2-4], chronic diseases monitoring [5], and depression detection [6], to cite a few. In this kind of application, the subject is continuously monitored, commonly by means of a sensor network. Each sensor in the network generates a stream of data which in turn is used to detect anomalies. The monitoring system, in these cases, is able to detect an abrupt change in the data stream, for example, an anomalous reading from the accelerometer might signal a fall [7]. Moreover, anomaly detection [8] plays a central role in realms such a intrusion/fraud detection [9,10], quality control in the Industry [11], public health breakouts [12], and climate change [13].

Statistical Process Control (SPC) [14], applied in industries for quality control, has faced the issue of abrupt change detection for a long time. The statistical approach is well suited when monitoring temporal data which follows a known probability distribution function $(p d f)$ and the parameters of the $p d f$ are also known before and after the change, which is the case of the cumulative sum algorithm, also know as CUSUM algorithm $[15,16]$.

In contrast with abrupt changes, progressive changes, or changes with some trend in the data stream, are more difficult to detect, especially if the data is randomly distributed, like in the case of ADL. This is the case for most older-specific illnesses, like cognitive decline, Alzheimer disease (AD), dementia or functional impairment in general [17-20].

Due to the importance of an early detection of cognitive decline in the older adults $[21-24]$ and to the rate at which the world population is aging [25], to develop techniques for early detecting changes in ADL, and to estimate the point in time where the change happened, is of great importance. This, in turn, can be integrated in already e-health systems to detect changes in continuously monitored older adults [26]. In this kind of systems the older adults are continuously monitored by means attached to the users' body and the collected data is sent using wireless communication to a server for later analysis. Analyzed data can provide insights in physical aspects of health, but also on psychological aspects of health as loneliness, social isolation life satisfaction and quality of life $[27,28]$ 
Our first hypothesis is that periodic ADLs can be modeled using circular normal distribution functions. Our second hypothesis is that abrupt changes in periodic ADLs modeled by circular normal distribution can be detected and the change point in time can be estimated by means of the methods used in SPC. Our third hypothesis is that the methods applied for abrupt change detection can be extended to detect changes in a circular normal distribution which at some point in time starts to follow a linear drift, and this result can be applied to detect a linear drift in ADLs.

Both cases are quite important when monitoring ADL of older adults. Abrupt change detection, when applied to ADL, might indicate that a change in the medication taken by an older adult has had some effect on his/her behaviour [29]. A linear trend detection in ADL might indicate the start of some cognitive decline, for example Alzheimer Disease [30,31].

Change detection in ADLs is of special interest in early detection of cognitive decay in older adults [32]. There exist evidences that circadian rhythm disruption is a symptom of neurodegeneration, which in turn changes ADLs patterns [3335]. Recently, a relationship has been suggested between sleep characteristics and cognitive decline in the older adults, emphasizing the fact that sleep and cognition are closely related [36-42]. The authors in [43] present a one year study with 77 older adults (21 mild AD, 27 moderate to severe AD and 29 control subjects). The results show that patients with moderate to severe AD have earlier bedtimes (9:45, $\sigma=1 \mathrm{~h} 31 \mathrm{~min})$ compared with patients with mild AD (10:45, $\sigma=1 \mathrm{~h} 36 \mathrm{~min})$ and control (11:30, $\sigma=58 \mathrm{~min})$. No differences regarding the rise time were reported, moderate to server $\operatorname{AD}(7: 15, \sigma=55 \mathrm{~min})$, mild $\mathrm{AD}(6: 55, \sigma=54 \mathrm{~min})$ and control subjects $(7: 00, \sigma=47 \mathrm{~min})$.

Furthermore, some works positively correlate the severity of the disorder and the severity of the cognitive impairment [32,44,30,31]. An early study by Stem, R. G. et al [45] on 183 patients (111 with $\mathrm{AD}$ and 72 non-AD) during 90 months showed a positive linear correlation between the scores of the AD patients on the Cognitive Subscale of the Alzheimer's Disease Assesment Scale and the assesment time within 24 months. In addition, this study also showed that the rate score followed a quadratic correlated with the score at the assessing time.

Time pattern followed by an older adult in out-of-home activities has been recently correlated with cognitive decay. A study including 85 independent older adults who live alone has stated that more hours spent outside the home was associated with better cognitive functions measured by the Clinical Dementia Rating [46]. Similar results was found out in [47].

Cognitive inspired techniques play an important role in Ambient Assisted Living for developing new systems which improve the quality of life of the citizens in general and the older adults in particular [48]. Activities of Daily Living recognition is a key aspect in such a systems which is mainly performed applying machine learning techniques [49] to data provided by an in-home sensor network [26]. Other novel techniques are based on analyzing the speech and emotional temperature [50] or the performance on handwriting/drawing tasks on digitizing tablets [51].

The contributions of our work are the following:

1. We model Activities of Daily Living (ADL) by means of circular normal probability distributions, and we asses its validity by performing experiments on two public data sets. 
2. We extend the CUSUM algorithm in order to detect a linear trend in a process following a circular normal distribution, and we use MLE to estimate the change point.

3. We perform extensive simulations in order to asses the validity of the presented approach in the two cases: abrupt change and linear drift change.

The rest of the paper is organised as follows: Section 2 presents the background in the fields of SPC, Human Activity Recognition (HAR), Circular Statistics, and Anomaly Detection on ADL. Section 3 shows how to model ADL by means of a circular normal distribution; this section also presents the CUSUM algorithm and how it is applied to detect abrupt changes in a process following a circular normal distribution; then, a contribution extending the CUSUM algorithm to detect linear trends is presented and applied to the case of a process following a circular normal distribution. Section 4 presents an extensive set of experiments performed to asses the validity of the presented method. Finally, Section 5 presents the main conclusions.

\section{Background}

In this section, SPC is first introduced, as the method presented in this paper relies on SPC techniques. Then, HAR is presented as the base problem of identifying ADL. Finally, some related works on the field of anomalies detection are reviewed.

\subsection{Statistical Process Control}

SPC is a main field for quality control in industrial processes. Since its introduction by Shewhart [52], SPC control charts have become one of the most used tools for change detection in industrial processes. Other extensively and successfully used control charts are the Cumulative Sum (CUSUM) introduced by Page $[15,16]$ and the Exponential Weighted Moving Average (EWMA) introduced by Roberts [53]. In general, the main objective of control charts is to detect, as soon as possible, abrupt changes in monitored processes [54].

After detecting an abrupt change, the next step is to estimate the time when the change has happened. Different approaches have been presented for change point estimation: neural networks [55-57], fuzzy sets [58-60,49,61] and the bayesian approach [62,63], among others. Nevertheless, most existing approaches are based on MLE [64-66].

In most cases, the detection algorithm and estimation method assume an abrupt change in the process. However, a more gradual change, such as a linear trend disturbance, may be important to consider [64-66]. The results in these last cases show that the performance and accuracy of the abrupt change methods are outperformed by those methods specifically designed for linear drift anomaly detection [64].

\subsection{Human Activity Recognition}

The goal of HAR is to find out the set of activities performed by a person in a time interval, based on some measured attributes. Formally: given a set of activity labels 
$A=\left\{A_{1}, \ldots, A_{i}\right\}$, a time interval $T=\left[t_{\text {start }}, t_{\text {end }}\right]$, and a set of $j$ time series, each one from a particular measured attribute $S=\left\{S_{1}, \ldots, S_{j}\right\}$ the goal is to find out the sequence of non overlapping activities $A=\left\langle A_{\left[t_{\text {start }}, t_{1}\right)}, A_{\left[t_{1}, t_{2}\right)}, \ldots, A_{\left[t_{k}, t_{\text {end }}\right]}\right\rangle$ based on the data on $S$ [67]. Some typical activities are: getting out of bed, eating, watching TV, bathing and going to bed, just to cite a few.

The measured attributes can be image recordings [68-70], binary sensors attached to doors, drawers and windows $[68,71]$ in the case of external sensors; and accelerometers, gyroscopes, temperature or heart rate sensors worn by the human being $[68,67]$.

In this work, it is assumed that the set of non overlapping activities and the time interval for each of them $A=\left\langle A_{\left[t_{\text {start }}, t_{1}\right)}, A_{\left[t_{1}, t_{2}\right)}, \ldots, A_{\left[t_{k}, t_{\text {end }}\right]}\right\rangle$ is already known. The goal of this paper is to detect changes in routine activities such as going to bed, based on its starting or ending time, since such changes in a person's behavior can indicate a decay in health.

\subsection{Circular statistics}

Directional statistics deals with data that represents directions. In the case of two dimensions, directional statistics is named circular statistics. A particular case is periodic data, for example, the arrival times of patients at a hospital [72], where each time in the clock face represents a direction.

Circular statistics has been used successfully for modeling human activity recognition [73,74], time patters in crime analysis [75], and analysis of social interactions and mood [76]. In all cited cases there is a clear periodicity of the studied phenomenon regarding the time; this periodicity might be a day, a week, or even a year or longer periods of time.

Different circular $p d f s$ have been used to model directional data. The circular Gumbel distribution has been used in [77] to model ADL. Also, the Wrapped Normal distributions has been used in $[78,72]$ to model ADL. The circular normal distribution, also known as von Mises distribution, has been extensively used to model directional data $[75,76,73,74]$.

\subsection{Anomaly detection}

Following [79], we define anomalous activities as events with the following properties: a) they occur rarely and, b) they have not been expected in advance.

These properties highlight two facts: a) it is difficult to find out HAR databases containing anomalous activities; b) it is also difficult to know the parameters that define an anomalous activity.

Several techniques have been used to detect anomalous activities. A two-phase pipeline process is used in [80]. In the first phase, normal activities are filtered out using a one-class Support Vector Machine addressed to reduce the false positive rate. The second phase uses an iterative procedure in which a Hidden Markov Model (HMM) of the initial activities is first created, and each new activity instance is then checked against the current model. Every new activity is thus classified using the trained HMM, and if its likelihood is below a given threshold, it is considered as anomalous, and a new class is created for it. 
A variant of the Hamming distance is used in [77] to measure the dissimilarity between two circular sequences of data. These sequences models ADL of the older adults at home as circular Gumbel distributions. The data was acquired during six months using a network of PIR motion sensors installed in a 80-year woman flat.

Probabilistic models including Naïve Bayes, Hidden Markov Model, Hidden Semi-Markov Models and Conditional Random Fields are compared in [71] using three datasets acquired in real scenarios. Data is provided by several sensors as reed switches, pressure mats, mercury contacts, passive infra-red and float sensors [81]. The presented results show that an increase in model complexity also improves the results.

The work in [82] also uses an HMM to detect anomalous activities, then exponential smoothing on histories of time series is used to predict future changes, and finally both outcomes are fused to detect anomalous activities. In the former two works, an activity willing to be classified as anomalous might consist on two consecutive activities in the time series being exchanged, but any abrupt change in the beginning or ending time of a single activity would not be detected as an anomalous behavior. On the contrary, the work presented in this paper is able to detect anomalous behaviors consisting on a delay in the beginning time of an activity.

A probabilistic anomalous detection method based on dynamic Bayesian networks is presented in [83]. Information provided by wearable sensors and location context is used to detect anomalous behaviors in spatial, timing, duration and sequences of ADLs.

A two-layered extension of the hidden semi-Markov model is used in [84] for modeling ADL based on the data provided by a set of cameras. This model, in turn, is used to detect anomalies in the duration of activities.

Machine Learning techniques are commonly used to detect anomalous behaviors. In particular [85] and [86] use Support Vector Machines (SVM) to build models based on normal behavior data to latter try to detect anomalous behaviors.

The authors in [87] use a semantic approximation for detecting anomalous behaviors of mild cognitive impairment. With the help of cognitive neuroscience researchers, both normal and anomalous activities are represented using a Web Ontology Language version 2 (OWL 2) ontology. Logic inference is used to find out anomalous behavior.

Infrared sensor and magnetic contacts in doors are used in [88] to locate an user in order to measure her circadian activity. After registering long periods of activity, a probabilistic model is built. Then, activities with high entropy are considered to be anomalous behaviors.

Information provided by binary sensors and its activation times are used in [89] to create regularity and duration scores. The authors use density based spatial clustering with noise on the scores to cluster activities. The cluster with the largest number of instances represents the normal behavior. 


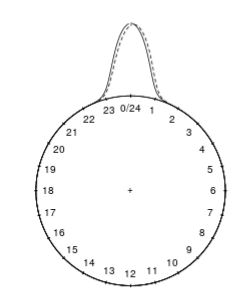

$\mu=0 \mathrm{~min}, \kappa=84.5(25 \mathrm{~min})$ $\mu=5 \mathrm{~min}, \kappa=84.5(25 \mathrm{~min})$

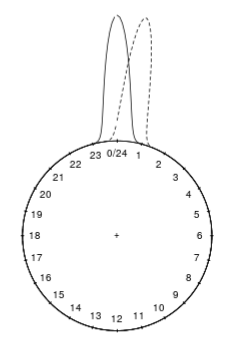

$\mu=0 \min , k=517(10 \mathrm{~min})$ $\mu=30 \min , \kappa=517(10 \mathrm{~min})$

Fig. 1 Examples of circular normal distribution functions. Solid lines show the circular normal distribution before an abrupt change, dashed plots show the circular normal distribution after a change. The case on the left shows a challenging to detect. On the contrary, the case on the right shows an easy case to detect.

\section{Methodology}

The circular distribution is introduced first in this section to model periodic events. Then abrupt change detection using the CUSUM algorithm is presented. After that, the main contribution of this work is presented: a novel method to detect a linear trend in a circular normal distribution.

\subsection{Activity modeling using the circular normal distribution}

The circular normal distribution, is a symmetric unimodal distribution describing circular data with periodical nature. This kind of data arise in a number of fields such as biology, geography, geology geophysics, medicine, meteorology and oceanography. Examples of such data include directions of wind and ocean currents, directional movement of animals in response to stimuli, and biorhythms [90], [88].

The probability density function of the circular normal distribution for a given angle $\phi$ is given by:

$$
f(\phi \mid \mu, \kappa)=\frac{e^{\kappa \cos (\phi-\mu)}}{2 \pi I_{0}(\kappa)}
$$

$\forall \phi \in \Re, \mu \in[0,2 \pi)$ and $\kappa \geq 0$. The $\mu$ parameter is known as the mean direction parameter, the $\kappa$ parameter is know as a concentration parameter, and $I_{0}(\kappa)$ is the modified Bessel function of order 0 defined by:

$$
I_{0}(\kappa)=\frac{1}{2 \pi} \int_{0}^{2 \pi} e^{\kappa \cos \varphi} d \varphi
$$

The variance of the circular distribution is given by: 
Table 1 Equivalences between some concentration parameters $\kappa$ and the squared root of the variance for a circular distribution.

\begin{tabular}{cc}
\hline$\kappa$ & $\sigma$ (in minutes) \\
\hline 33 & 40 \\
58 & 30 \\
84.5 & 25 \\
131 & 20 \\
233 & 15 \\
517 & 10 \\
\hline
\end{tabular}

$$
\sigma^{2}=1-\frac{I_{1}(\kappa)}{I_{0}(\kappa)}
$$

If the value of mean direction $\mu \in[0,2 \pi)$ is taken as time units in the range [0, 24) hours, the equivalence between the concentration parameter $\kappa$ and the squared root of the variance $\sigma$ can be extracted using (1). Table 1 shows some equivalent values used in the experiments presented in Section 4. In the remainder of this work the parameter $\sigma$, in minutes, will be used instead of $\kappa$ because it can be interpreted as the dispersion of the data, for example the dispersion time for a person's wake up time. Fig. 1 shows some circular normal probability distribution functions plots. Note that parameter $\kappa$ is related with the dispersion of the data, the bigger $\kappa$ the lower dispersion of the data.

For a set of samples $\left\{\phi_{i}\right\}$ with $i \in[1, N]$, the MLE for $\mu$ and $\kappa$ are:

$$
\begin{aligned}
& \hat{\mu}=\tan ^{-1}\left(\frac{\sum_{i=1}^{N} \sin \phi_{i}}{\sum_{i=1}^{N} \cos \phi_{i}}\right) \\
& \hat{\kappa}=\frac{1}{n}\left[\left(\sum_{i=1}^{N} \cos \phi_{i}\right)^{2}+\left(\sum_{i=1}^{N} \sin \phi_{i}\right)^{2}\right]
\end{aligned}
$$

These two expressions can be used to estimate the parameters $\mu$ and $\kappa$ from a set of data.

Two public data sets from the CASAS project have been used to assess the validity of using the circular normal probability distribution for modeling ADLs. The first data set is called Milan [91]. The data set is annotated with ADL and was acquired between $16 / 10 / 2009$ and $6 / 1 / 2010$ by an older adult leaving alone in an apartment. The second data set is called Aruba [92]. The data set is annotated with ADL and was acquired between 4/11/2010 and 11/6/2011 by an older adult leaving alone in a apartment.

In order to test if ADL can be modeled by circular normal distributions, six ADL were selected from each data set, and there were adjusted to a mixtures of circular normal distributions using the $\mathrm{R}$ library movMF [93]. The KullbackLeibler divergence [94] was used to calculate the divergence between the empirical probability density function and the fitted probability density function. Results are shown on Tables 2 and 3. Figures 2 and 3 show the data for the Milan and Aruba data sets using a rose diagram; the solid lines show the empirical density from the data, the dashed lines show the density for the fitted mixtures of circular 
Table 2 Mila data set. The name of the ADLs are the same as used in the public data set. \#pfs is the number of circular normal distributions used to fit the data. KL is the KL-divergence between the empirical density and the density of the fitted data. Ex: $\operatorname{Time}(\sigma)$ stands for an example of the time of the activity and its fitted $\sigma$ in minutes.

\begin{tabular}{ccccc}
\hline ADL & samples & \#pdfs & KL & Ex: Time $(\sigma)$ \\
\hline Sleep begin & 156 & 8 & 0.0252 & $22: 17(38.05)$ \\
Sleep end & 156 & 8 & 0.0120 & $08: 14(17.28)$ \\
Bed to toilet begin & 88 & 8 & 0.0072 & $02: 11(13: 52)$ \\
Bed to toilet end & 88 & 8 & 0.0045 & $02: 13(13.40)$ \\
Leave home begin & 214 & 8 & 0.0145 & $17: 16(30.95)$ \\
Leave home end & 214 & 8 & 0.0059 & $17: 22(31.00)$ \\
\hline
\end{tabular}

Table 3 Aruba data set. The name of the ADLs are the same as used in the public data set. \#pfs is the number of circular normal distributions used to fit the data. KL is the KLdivergence between the empirical density and the density of the fitted data. Ex: Time $(\sigma)$ stands for an example of the time of the activity and its fitted $\sigma$ in minutes.

\begin{tabular}{ccccc}
\hline ADL & samples & \#pdfs & KL & Ex: Time $(\sigma)$ \\
\hline Sleep begin & 401 & 6 & 0.0182 & $00: 12(24.52)$ \\
Sleep end & 401 & 8 & 0.0260 & $05: 45(26.97)$ \\
Bed to toilet begin & 157 & 7 & 0.0063 & $00: 34(8.85)$ \\
Bed to toilet end & 157 & 8 & 0.0036 & $00: 36(9.23)$ \\
Leave home begin & 431 & 9 & 0.0093 & $14: 48(8.80)$ \\
Enter home begin & 431 & 9 & 0.0051 & $15: 01(36.69)$ \\
\hline
\end{tabular}

normal distributions. The radial segments at these Figures correspond with the example times shown in Tables 2 and 3. The KL-divergence of all analyzed data is less than 0.03, and the maximum of the lobes for the activities are near of the density of real data. The analysis of these data suggest that a mixture of circular normal distributions might be use to feasibly model ADLs.

In addition to our own data analysis, the data plots presented in [36] and [29] also suggest that ADLs (going to the bathroom, going to the bedroom, going out of home) could be modeled by a mixture of circular normal distribution.

\subsection{Abrupt change detection and change point estimation}

The CUSUM algorithm is a quality control method which was first proposed in [16] as a continuous inspection scheme to detect changes in a sequence of independent random variables following some known probability function.

If it is assumed that a signal can be modeled as a discrete random signal $\mathcal{Y}$, with independent and identically distributed samples, the $p d f$ of each sample is given by $p_{\theta}\left(y_{i}\right)$, where $\theta$ is a deterministic parameter. The occurrence of an event is modeled by an instantaneous change in $\theta$, so that $\theta=\theta_{1}$ before the event at $i=\tau$ and $\theta=\theta_{2}$ when $i \geq \tau$. Thus, the two possible hypotheses are:

$$
\begin{aligned}
& \boldsymbol{H}_{1}: \theta=\theta_{1}, \quad \text { No change has happened. } \\
& \boldsymbol{H}_{2}: \theta=\theta_{2}, \quad \text { A change has happened. }
\end{aligned}
$$

The $p d f$ of the signal $\mathcal{Y}$ observed between the initial sample $y_{1}$ and the current sample $y_{N}$ can take two forms depending on the above hypotheses. Under the ' $n o$ change' hypothesis, also know as the in-control process, $\boldsymbol{H}_{1}$, the $p d f$ is: 
Sleep begin

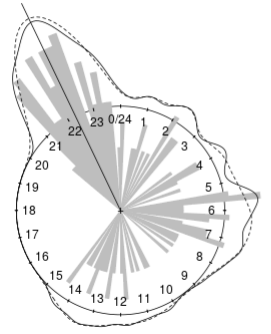

Sleep end

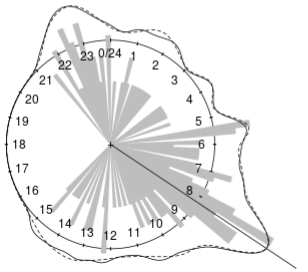

Bed to toilet begin

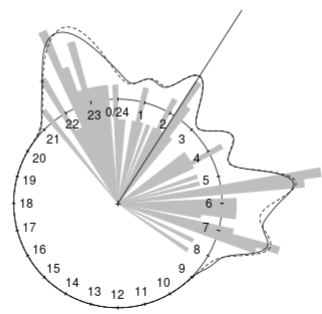

Bed to toilet end

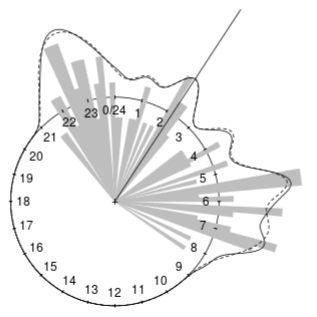

Leave home begin

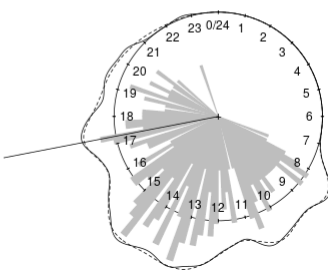

Leave home end

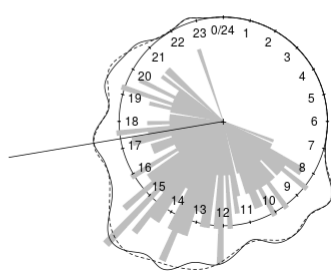

Fig. 2 Activities in the Milan data set. Rose diagrams show the data at intervals of 10 minutes. The solid line shows the density of the data. The dashed line shows the density of the fitted data. The solid segment at each activity is the time for the fitted circular normal distribution showed in Table 2 .

$$
p_{\theta_{1} \mid \boldsymbol{H}_{1}}=\prod_{i=1}^{N} p_{\theta_{1}}\left(y_{i}\right) .
$$

On the other hand, under the 'change' hypothesis, also know as the out-of control process, $\boldsymbol{H}_{2}$ the $p d f$ is:

$$
p_{\theta_{2} \mid \boldsymbol{H}_{2}}=\prod_{i=1}^{\tau-1} p_{\theta_{1}}\left(y_{i}\right) \prod_{i=\tau}^{N} p_{\theta_{2}}\left(y_{i}\right) .
$$

With these assumptions, the CUSUM algorithm defines two quantities, the cumulative sum:

$$
S_{\tau}^{N}=\sum_{i=\tau}^{N} \ln \frac{p_{\theta_{2}}\left(y_{i}\right)}{p_{\theta_{1}}\left(y_{i}\right)}=\sum_{i=\tau}^{N} s_{i}
$$

where $s_{i}=\ln \frac{p_{\theta_{2}}\left(y_{i}\right)}{p_{\theta_{1}}\left(y_{i}\right)}$ is defined as the instantaneous log-likelihood; and the decision function:

$$
g_{N}=\max _{1 \leq \tau \leq N} S_{\tau}^{N}
$$




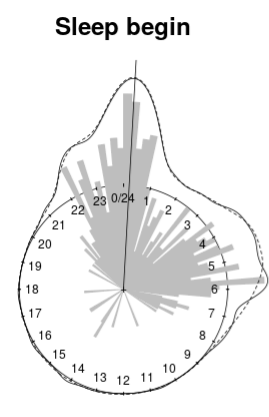

Sleep end

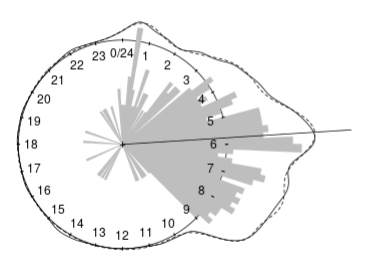

Bed to toilet begin

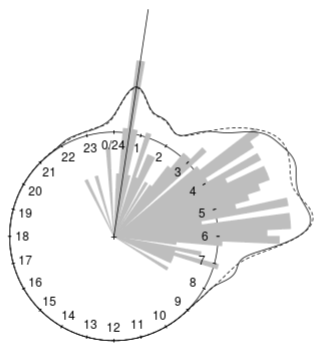

Bed to toilet end

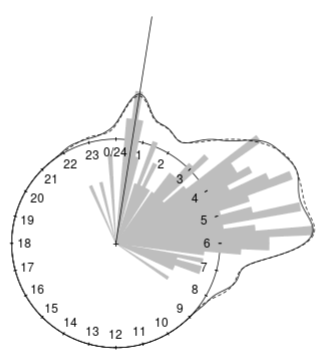

Leave home begin

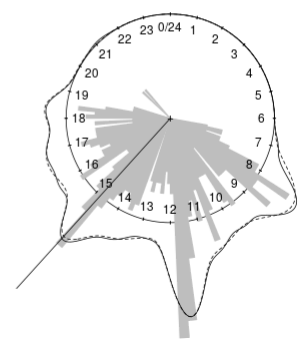

Enter home begin

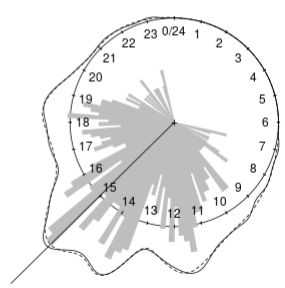

Fig. 3 Activities in the Aruba data set. Rose diagrams show the data at intervals of 10 minutes. The solid line shows the density of the data. The dashed line shows the density of the fitted data. The solid segment at each activity is the time for the fitted circular normal distribution showed in Table 3 .

Given a user-defined threshold $h$, if the decision function $g_{N}<h$ the hypothesis $\boldsymbol{H}_{1}$ is true and so no change has been produced. On the contrary, if the decision function $g_{N}>h$ the hypothesis $\boldsymbol{H}_{2}$ becomes true and so it is assumed that a change has happened. In the latter case, Maximum Likelihood can be used to find an estimate of the change point considering (3):

$$
\hat{\tau}=\arg \max _{1 \leq \tau \leq N} S_{\tau}^{N}
$$

Equations 2 and 3 can be rewritten in a recursive form: $S_{\tau}^{N}=S_{\tau}^{N-1}+s_{N}$ and $g_{N}=\left\{g_{N-1}+s_{N}\right\}^{+}$where $\{z\}^{+}=\sup (0, z)$. These recursive equations are convenient to evaluate the cumulative sum and decision function as data is produced, as in the case of ADL monitoring, and to raise an alarm in case an anomaly was detected; for example, an abrupt change in a monitorized person's wake up time over several consecutive days would trigger an alarm.

If we assume two processes described by a circular normal distribution $f\left(\phi \mid \mu_{1}, \kappa\right)$ and $f\left(\phi \mid \mu_{2}, \kappa\right)$ with same concentration parameter $\kappa$ but different mean direction parameter $\mu_{1}$ and $\mu_{2}$, the instantaneous log-likelihood can be written as: 


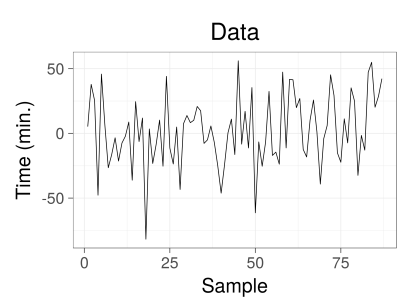

Data

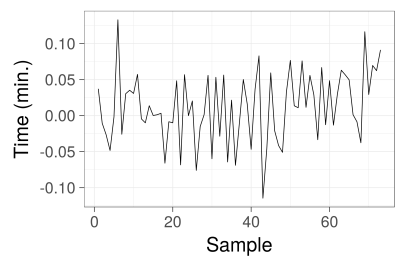

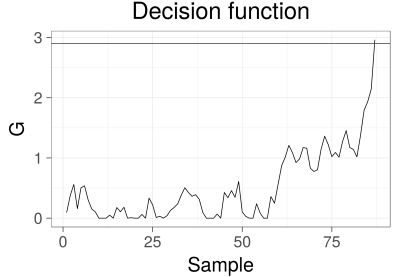

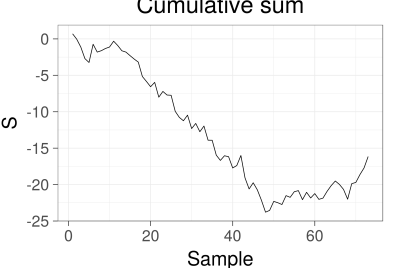

Decision function

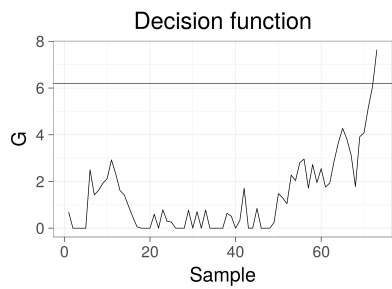

Fig. 4 Data (left), cumulative sum (middle), and decision function (right) for two sets of temporal data following a circular normal distribution. The change point was set to $\tau=50$ in both experiments. The plots on the first row were generated with $\mu_{1}=0, \mu_{2}=5 \mathrm{~min}$., $\sigma=$ 25 min. and $h=2.9$ (horizontal line). The plots on the second row were generated with $\mu_{1}=0, \mu_{2}=10 \min ., \sigma=10 \mathrm{~min}$. and $h=6.2$.

$$
\begin{aligned}
s_{i}=\ln \frac{f\left(\phi_{i} \mid \mu_{2}, \kappa\right)}{f\left(\phi_{i} \mid \mu_{1}, \kappa\right)}= & \kappa\left[\cos \phi_{i}\left(\cos \mu_{2}-\cos \mu_{1}\right)\right. \\
& \left.+\sin \phi_{i}\left(\sin \mu_{2}-\sin \mu_{1}\right)\right]
\end{aligned}
$$

An MLE for $s_{i}$ before the change point $(i<\tau)$ is proportional to:

$$
\hat{s_{i}}\left(\hat{\phi}_{i}=\mu_{1}\right) \propto \cos \left(\mu_{1}-\mu_{2}\right)-1 \leq 0
$$

In the same way, an MLE for $s_{i}$ after the change point $(i \geq \tau)$ is proportional to:

$$
\hat{s_{i}}\left(\hat{\phi}_{i}=\mu_{2}\right) \propto 1-\cos \left(\mu_{1}-\mu_{2}\right) \geq 0
$$

Regarding to $S_{i}$, it is a sum of negative values before de change point (4), and of positive values after the change point (5), so $S_{i}$ will have a local minimum in the interval $[1, N]$.

In summary, the most likely value for the instantaneous log-likelihood before the change point is less than zero. On the contrary, the most likely value for the instantaneous log-likelihood after the change point is great than zero, so the cumulative sum will decrease before the change point and will increase after the change point. Fig. 4 shows a set of plots with an example for the cumulative sum $S_{i}$ and decision function $g_{i}$ for processes following a circular normal distribution, when an abrupt change was introduced at time $\tau=50$. 


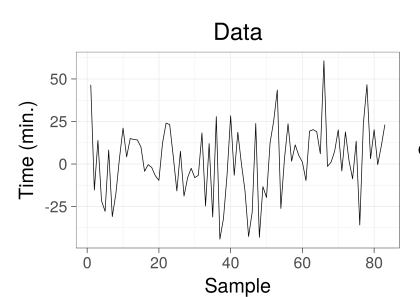

Data

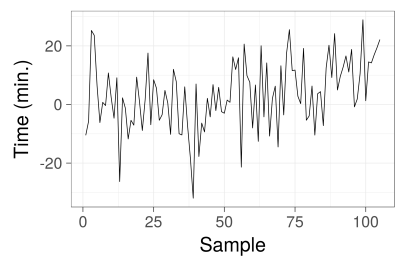

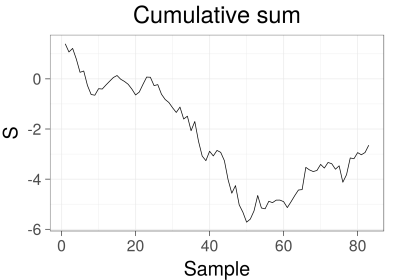

Cumulative sum

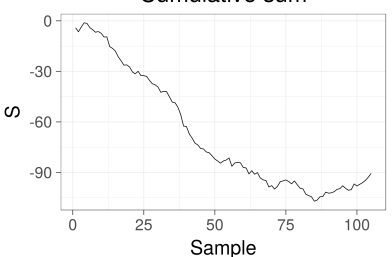

Decision function

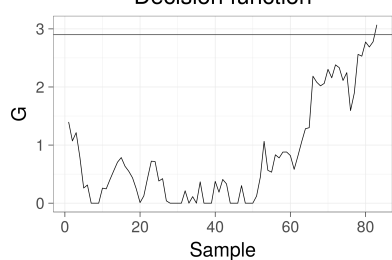

Decision function

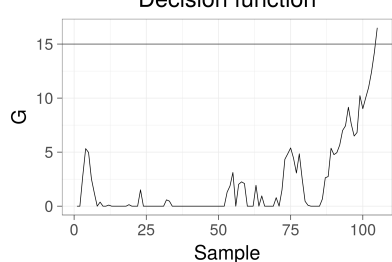

Fig. 5 Data (left), cumulative sum (middle), and decision function (right) for two sets of temporal data following a circular normal distribution. The change point was set to $\tau=$ 50 in both experiments. The plots on the first row were generated with $\sigma=25 \mathrm{~min}$., $\beta=$ 0.25 min. per day and $h=2.9$ (horizontal line). The plots on the second row were generated with $\sigma=10$ min., $\beta=0.25$ min. per day and $h=6.2$.

\subsection{Linear trend detection and change point estimation}

Let's assume an elderly person living alone and continuously monitored at her own home $[36,95]$. The monitoring system detects a drift in her daily routines, for example time to bed, wake up time and time to go out of home. Health practitioners decide her to answer the Mini-Mental State Examination, and the results suggest that she is starting a cognitive decay. Some time later, she is diagnosed with $\mathrm{AD}$ and starts to take medication under medical prescription. Again, some time later the monitoring system detects another drift in her daily routine which might be related with de medication provided by health practitioners [29]. This scenario describes an hypothetical case for the main contribution of our work, to detect such a linear drift in the data for ADL.

If a process starts to follow a linear drift at event $i_{0}=\tau$ at rate $\beta$, it can be modeled as:

$$
\begin{aligned}
& \theta(t \leq \tau)=\theta_{1} \\
& \theta(t>\tau)=\theta_{2}=\theta_{1}+(i-\tau) \beta
\end{aligned}
$$

Note that the drift rate $\beta$ is unknown, so some heuristic should be used to detect that the process has started to follow a linear drift.

To detect that the process has started a linear drift, the following pair of hypothesis is used:

$$
\begin{gathered}
\boldsymbol{H}_{1}: \theta=\theta_{1}, \quad \text { No change has happened. } \\
\boldsymbol{H}_{2}: \theta=\theta_{1}+\delta, \quad \text { A change has happened. }
\end{gathered}
$$


where $\boldsymbol{H}_{1}$ is the no change hypothesis, and $\boldsymbol{H}_{2}$ is the change hypothesis. If $\delta>0$ the change happened after time $\theta$, on the contrary, if $\delta<0$ the change happened before time $\theta$. Without loss of generality, it will be assumed $\delta>0$ in the remainder of this work. Hereafter, the parameter $\delta$ will be called the detector time.

Particularizing for the circular normal distribution, without loss of generality, it can be assumed that $\mu_{1}=0$ and given that $\mu_{2}=\delta$, the instantaneous loglikelihood in (4) becomes:

$$
\hat{s}_{i}=\kappa\left[\cos \left(\phi_{i}\right)(\cos (\delta)-1)+\sin \left(\phi_{i}\right) \sin (\delta)\right]
$$

A MLE for $s_{i}$ before the change is proportional to:

$$
\hat{s}_{i} \propto \cos (\delta)-1
$$

so the MLE of the instantaneous log-likelihood is negative before the change, and after the change is proportional to:

$$
\hat{s}_{i} \propto \cos (i \beta)(\cos (\delta)-1)+\sin (i \beta) \sin (\delta)
$$

this last expression is negative when $\tan (i \beta)<\frac{1-\cos (\delta)}{\sin (\delta)}$ and positive when $\tan (i \beta)>$ $\frac{1-\cos (\delta)}{\sin (\delta)}$. So at point $\tan (i \beta)=\frac{1-\cos (\delta)}{\sin (\delta)}$ the cumulative sum, and the decision function start to increase. Finally, the decision function will reach a given threshold and it can be considered that a change has happened. Fig. 5 shows a set of plots for the cumulative sum $S_{i}$ an the decision function $g_{i}$ for two processed following a circular normal distribution when a linear drift was introduced at time $\tau=50$.

The next step after detecting the change is to estimate the rate of the process after the change. In our case, once the change has been detected we define a series of hypothesis $\boldsymbol{H}_{n}$ at regular intervals ahead of the current value of $\theta\left(\theta_{1, i>\tau}\right)$, and we use the classic CUSUM algorithm for each pair of consecutive hypothesis.

Formally, this is expressed in the following way. If we assume a linear trend in the process, without loss of generality, we can define a set of $W$ pairs of hypothesis equally spaced $(\delta)$ in the parameter $\theta$ :

$$
\begin{gathered}
\begin{cases}\boldsymbol{H}_{1}: \theta=\theta_{1}, \quad \text { No change has been produced. } \\
\boldsymbol{H}_{2}: \theta=\theta_{1}+\delta, \quad \text { A change has been produced. }\end{cases} \\
\begin{cases}\boldsymbol{H}_{2}: \theta=\theta_{1}+\delta, & \text { No change has been produced. } \\
\boldsymbol{H}_{3}: \theta=\theta_{1}+2 \delta, & \text { A change has been produced. }\end{cases} \\
\left\{\begin{array}{l}
\boldsymbol{H}_{W} \quad: \theta=\theta_{1}+(W-1) \delta, \quad \text { No change has been produced } \\
\boldsymbol{H}_{W+1}: \theta=\theta_{1}+W \delta, \quad \text { A change has been produced. }
\end{array}\right.
\end{gathered}
$$

The cumulative sum algorithm can be applied to each pair of consecutive hypothesis, $\boldsymbol{H}_{w}$ and $\boldsymbol{H}_{w+1}$ with $w \in[1, W]$. The change point for each pair of hypothesis can be estimated by: 


$$
\begin{aligned}
S_{\tau+1, w}^{N} & =\sum_{i=\tau}^{N} \ln \frac{p_{\theta_{1}+w \delta}\left(X_{i}\right)}{p_{\theta_{1}+(w-1) \delta}\left(X_{i}\right)} \\
\hat{\tau}_{w} & =S_{1, w}^{N}-\arg \min _{1 \leq \tau \leq N} S_{1, w}^{\tau}
\end{aligned}
$$

At any time the cumulative sum $S_{\tau+1, w}^{T}$ reaches a minimum it means that hypothesis $\boldsymbol{H}_{w+1}$ becomes valid and $\boldsymbol{H}_{w}$ is no longer valid. This minimum is reached, for the pair of hypothesis $\boldsymbol{H}_{w}$ and $\boldsymbol{H}_{w+1}$, at $\hat{\tau}_{w}$, as stated by (7). As the out-of control process evolves at rate $\beta$ over time, the minima $\hat{\tau}_{w}$ should evolve with the same rate, so we can write:

$$
\frac{\partial \hat{\tau}_{w}}{\partial w}=\frac{\partial \theta_{1, i>\tau}}{\partial i}=\hat{\beta}
$$

Equation 8 is the key point of our proposed algorithm, if we are able to estimate the linear trend rate of the minima for a set of pairs of hypothesis, we will be able to measure the linear trend of the process after the change.

\subsection{Simulated data generation}

Random data sets were generated before the change point $(\tau)$ using a circular normal distribution with constant mean direction $\mu_{1}$ and concentration parameter $\kappa$, and with a white noise added to it $N(0, \epsilon)$ :

$$
y_{i}(i \leq \tau) \rightarrow f\left(\phi \mid \mu_{1}, \kappa\right)+N(0, \sigma) ; \mu_{1}=\text { cte } ; \ll \mu_{1}
$$

after the change point, the mean direction $\mu_{2}$ parameter of the circular normal distribution starts to follow a linear drift with rate $\beta$, the concentration parameter $\kappa$ does not change:

$$
y_{i}(i>\tau) \rightarrow f\left(\phi \mid \mu_{2}, \kappa\right)+N(0, \sigma) ; \mu_{2}=\mu_{1}+(i-\tau) \beta ; \sigma \ll \mu_{1}
$$

Figure 5 shows, on the left, some realizations of simulated data.

\section{Results}

As stated in Section 2.4, anomalous activities occur rarely, and they are not expected in advance. These implies that it is difficult to find out any public ADL database containing anomalous behaviors, and so it is indeed difficult to figure out the rate for anomalous behaviors in ADLs. For this reason, in order to asses the validity of the presented method, anomalous behavior data were simulated, as done in related approaches (e.g. [88] and [82]). The simulated data were generated following the expression presented in Section 3.4.

This section presents the results of applying our proposal for using the CUSUM algorithm to a process following a circular normal distribution, according to the procedures described above. The performance in abrupt change detection is first presented and discussed, followed by the performance in linear trend detection. 
Table 4 Results for abrupt changes experiments. $\mu_{a f t e r}$ and $\sigma$ are set in minutes. The Mean Run Length (MRL) and $\overline{\hat{\tau}}$ are in days. False Alarms, Change Fails and $\hat{\tau}$ are in units as number of experiments. Success is the percentage of successful experiments.

\begin{tabular}{|c|c|c|c|c|c|c|c|c|}
\hline$\sigma$ & $\mu_{a f t e r}$ & $h$ & MRL & $\overline{\hat{\tau}}$ & $\begin{array}{c}\text { False } \\
\text { Alarms }\end{array}$ & $\begin{array}{c}\text { Change } \\
\text { Fails }\end{array}$ & $\begin{array}{c}\hat{\tau} \\
\text { Fails }\end{array}$ & Success \\
\hline 40 & 5 & 1.2 & $90.76 \pm 0.31$ & $51.82 \pm 0.35$ & 1426 & 1760 & 134 & 66.80 \\
\hline 40 & 10 & 2.5 & $90.01 \pm 0.26$ & $51.40 \pm 0.27$ & 681 & 969 & 88 & 82.62 \\
\hline 40 & 15 & 3.7 & $85.84 \pm 0.22$ & $51.22 \pm 0.21$ & 351 & 361 & 34 & 92.54 \\
\hline 40 & 20 & 5.2 & $83.25 \pm 0.19$ & $50.44 \pm 0.16$ & 106 & 107 & 20 & 97.67 \\
\hline 40 & 25 & 6.7 & $80.01 \pm 0.16$ & $50.09 \pm 0.12$ & 31 & 32 & 5 & 99.32 \\
\hline 40 & 30 & 9.1 & $79.71 \pm 0.14$ & $50.04 \pm 0.09$ & 0 & 5 & 2 & 99.93 \\
\hline 30 & 5 & 1.5 & $88.78 \pm 0.29$ & $52.90 \pm 0.32$ & 1565 & 1116 & 90 & 72.29 \\
\hline 30 & 10 & 3.4 & $88.79 \pm 0.24$ & $51.27 \pm 0.23$ & 392 & 593 & 44 & 89.71 \\
\hline 30 & 15 & 5.2 & $83.08 \pm 0.19$ & $50.61 \pm 0.16$ & 93 & 125 & 24 & 97.58 \\
\hline 30 & 20 & 7.7 & $80.84 \pm 0.15$ & $50.14 \pm 0.11$ & 14 & 20 & 6 & 99.60 \\
\hline 30 & 25 & 9.4 & $75.01 \pm 0.11$ & $50.00 \pm 0.07$ & 2 & 0 & 0 & 99.98 \\
\hline 30 & 30 & 10.4 & $69.71 \pm 0.09$ & $49.95 \pm 0.05$ & 0 & 0 & 0 & 100.0 \\
\hline 25 & 5 & 1.9 & $88.85 \pm 0.28$ & $51.76 \pm 0.30$ & 1165 & 1093 & 83 & 76.59 \\
\hline 25 & 10 & 4.1 & $85.49 \pm 0.21$ & $50.94 \pm 0.19$ & 232 & 365 & 34 & 93.78 \\
\hline 25 & 15 & 6.8 & $82.35 \pm 0.17$ & $50.18 \pm 0.12$ & 22 & 57 & 6 & 99.15 \\
\hline 25 & 20 & 9.8 & $77.97 \pm 0.12$ & $50.04 \pm 0.08$ & 0 & 4 & 1 & 99.95 \\
\hline 25 & 25 & 10.4 & $69.44 \pm 0.09$ & $49.98 \pm 0.05$ & 0 & 0 & 0 & 100.00 \\
\hline 25 & 30 & 10.7 & $63.95 \pm 0.06$ & $50.01 \pm 0.04$ & 0 & 0 & 0 & 100.00 \\
\hline 20 & 5 & 2.4 & $88.13 \pm 0.26$ & $51.63 \pm 0.27$ & 774 & 891 & 83 & 82.52 \\
\hline 20 & 10 & 5.1 & $82.35 \pm 0.18$ & $50.62 \pm 0.15$ & 96 & 120 & 26 & 97.58 \\
\hline 20 & 15 & 8.2 & $75.97 \pm 0.13$ & $49.94 \pm 0.09$ & 5 & 5 & 0 & 99.90 \\
\hline 20 & 20 & 11.4 & $71.60 \pm 0.09$ & $50.01 \pm 0.05$ & 0 & 0 & 0 & 100.00 \\
\hline 20 & 25 & 10.4 & $62.52 \pm 0.06$ & $50.00 \pm 0.03$ & 0 & 0 & 0 & 100.00 \\
\hline 20 & 30 & 10.5 & $58.78 \pm 0.04$ & $50.02 \pm 0.02$ & 0 & 0 & 0 & 100.00 \\
\hline 15 & 5 & 3.4 & $87.42 \pm 0.22$ & $50.57 \pm 0.21$ & 331 & 582 & 74 & 90.13 \\
\hline 15 & 10 & 7.6 & $80.15 \pm 0.15$ & $50.12 \pm 0.10$ & 10 & 20 & 2 & 99.68 \\
\hline 15 & 15 & 10.1 & $68.81 \pm 0.09$ & $49.98 \pm 0.05$ & 0 & 0 & 0 & 100.00 \\
\hline 15 & 20 & 10.3 & $60.94 \pm 0.05$ & $50.01 \pm 0.03$ & 0 & 0 & 0 & 100.00 \\
\hline 15 & 25 & 10.9 & $57.38 \pm 0.03$ & $49.99 \pm 0.02$ & 0 & 0 & 0 & 100.00 \\
\hline 15 & 30 & 10.5 & $54.92 \pm 0.02$ & $50.00 \pm 0.01$ & 0 & 0 & 0 & 100.00 \\
\hline 10 & 5 & 5.4 & $84.67 \pm 0.19$ & $50.61 \pm 0.16$ & 90 & 152 & 23 & 97.35 \\
\hline 10 & 10 & 10.2 & $69.25 \pm 0.09$ & $50.06 \pm 0.05$ & 0 & 0 & 0 & 100.00 \\
\hline 10 & 15 & 10.3 & $58.80 \pm 0.04$ & $49.98 \pm 0.02$ & 0 & 0 & 0 & 100.00 \\
\hline 10 & 20 & 11.5 & $55.48 \pm 0.02$ & $50.00 \pm 0.01$ & 0 & 0 & 0 & 100.00 \\
\hline 10 & 25 & 11.7 & $53.45 \pm 0.02$ & $50.00 \pm 0.01$ & 0 & 0 & 0 & 100.00 \\
\hline 10 & 30 & 10.5 & $52.02 \pm 0.01$ & $50.00 \pm 0.01$ & 0 & 0 & 0 & 100.00 \\
\hline
\end{tabular}

The $\sigma$ values for experimentation where chosen to be in accordance with those data extracted from the experimentation with real data sets presented in Section 3.1 (Tables 2 and 3) which ranges between $13.40 \leq \sigma \leq 38.05$ minutes for the Milan data set, and between $8.80 \leq \sigma \leq 36.69$ minutes for the Aruba data set.

4.1 Abrupt change detection and change point estimation in a process following a circular normal distribution

The CUSUM algorithm was developed to estimate the change point in time when a process following a known $p d f$ defined by some parameters, suddenly changes the value of one of its parameters. In the case of circular normal $p d f$ such parameter is $\mu$, and it is assumed that $\kappa$ is unchanged. Having into account the periodicity 

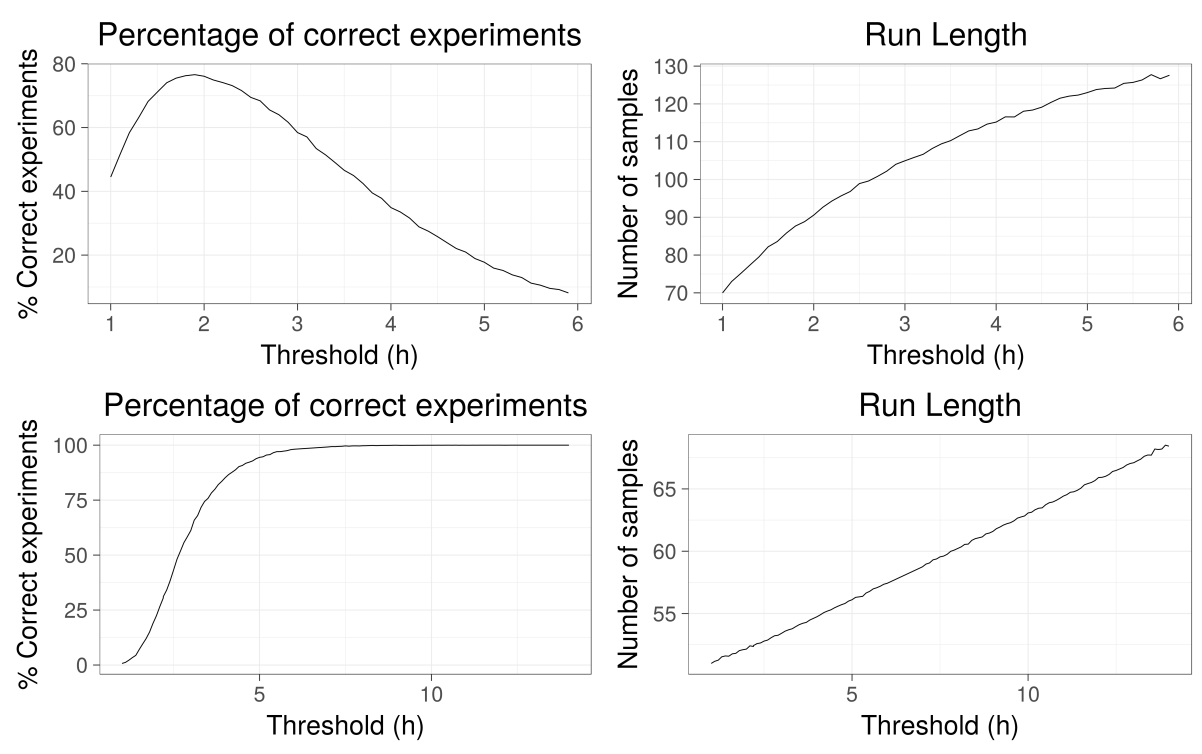

Fig. 6 Percentage of successful experiments as a function of decision function threshold (left). MRL as a function of decision function threshold (right). For this particular experiment $\sigma=25$ min.. For the first row $\mu_{a f t e r}=5$ min.. For the second row $\mu_{a f t e r}=30 \mathrm{~min}$. .

of the circular normal distribution, without loss of generality it can be assumed that $\mu=0$, in the case of the in-control process.

The first set of experiments were performed to asses the validity of the CUSUM algorithm to detect a change in a time series when a sudden change happens. As mentioned before, a case example is a sudden change in a person's wake up time due, for instance, to the beginning of a cold.

For each experiment 50 samples following a circular normal distribution were generated with parameter $\mu_{\text {before }}=0$, and given as basis that 24 hours are $2 \pi$ radians. The change point in time was set at sample 50th in all cases. After the change, 100 new samples following a circular normal distribution with parameter $\mu_{\text {after }}$ ranging from 5 minutes ( 0.022 radians) to 30 minutes ( 0.131 radians) were generated. As it was previously stated, parameter $\kappa$, and equivalently $\sigma$, was unchanged. For each combination of parameters, the experiment was repeated 10,000 times. The value for the threshold $h$ must be carefully set: if the value for the threshold is too low, a great number of false alarms could be detected; on the opposite, if the value for the threshold is too high, the decision function could never reach it. Fig. 6 shows how the percentage of successful experiments and the Mean Run Length (MRL) depends on the value of the decision function threshold $(h)$. An experiment is considered successful if the CUSUM algorithm is able at providing an estimate of the change point $\hat{\tau}$. Table 4 shows the experimental results for various combination of $\sigma$ (note the equivalence between $\sigma$ and $\kappa$ in Table 1 ) and $\mu_{\text {after }}$, each rows presents the results when the maximum number of successful experiments was reached (column labelled with Success). The MRL is the interval between the experiment start and the change detection. $\overline{\hat{\tau}}$ is the mean of the estimated change point. A False Alarms happens when a change is detected 


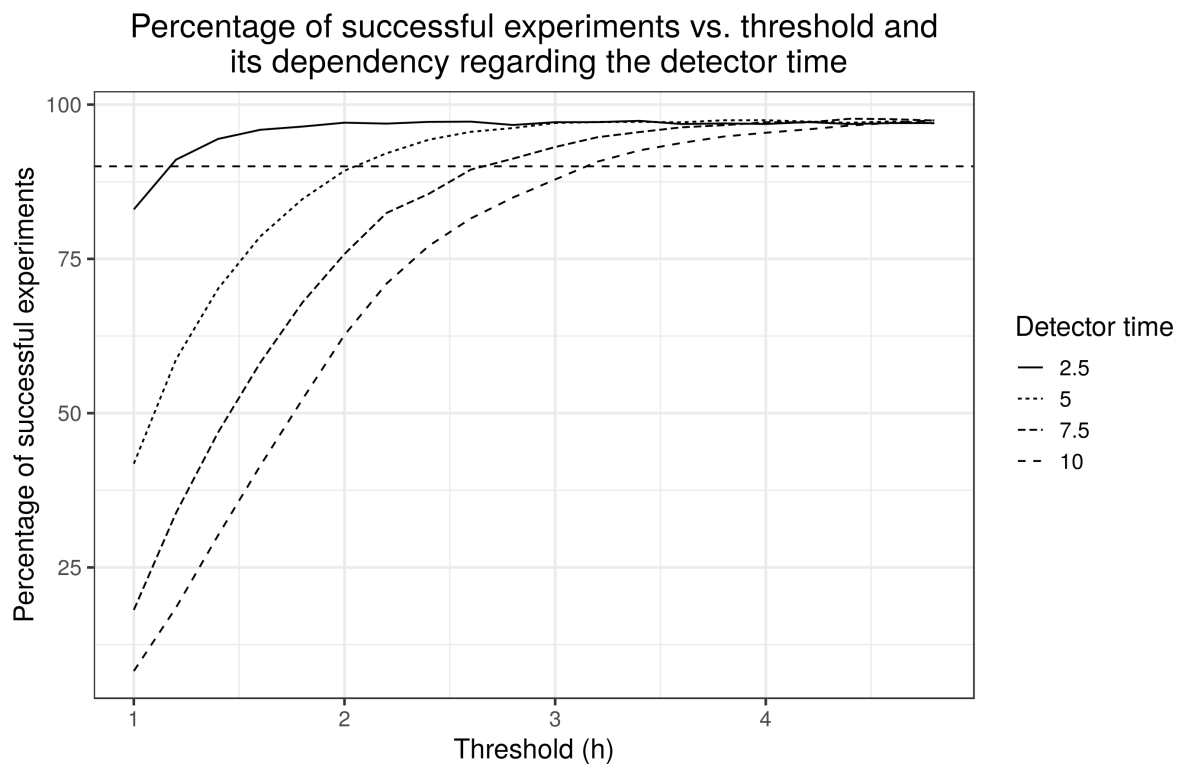

Fig. 7 Percentage of successful experiments vs. threshold for different detector times $\delta$ in minutes. $\sigma=25 \mathrm{~min}$. for all experiments. The dashed line is for $90 \%$ of successful experiments.

before the real change has happened, namely when $\hat{\tau}<\tau$. Change Fails is the number changes that were not detected by the algorithm. $\hat{\tau}$ Fail happens when a change is correctly detected but the algorithm is unable at estimating the change point $\hat{\tau}$. Finally, Success experiments is the percentage of experiments which were able at estimating the change point $\hat{\tau}$.

In all experiments, the mean estimated change point $\overline{\hat{\tau}}$ is quite close to the real value $\tau=50$. The differences between the real value for the change point $\tau$ and its estimated value $\overline{\hat{\tau}}$ is within the range $0 \leq\|\tau-\overline{\hat{\tau}}\| \leq 1.82 \pm 0.35$ for all performed experiments. The biggest difference corresponds to $\sigma=40 \mathrm{~min}$. and $\mu_{\text {after }}=5$ min., which is the most challenging case tested, since there is a high variability in the data $(\sigma=40 \mathrm{~min}$.), and the abrupt change in the mean $\mu$ is only 5 minutes. In the case example presented above, if a person's wake up time would be 7:00 a.m. with a dispersion of 40 minutes, and she suddenly change her wake up time 5 minutes, namely 7:05 a.m., the CUSUM algorithm might detect the change after an average of 41 days, and estimate the day of the change with an error of only 2 days.

For each pair of values $(\mu, \sigma)$ the value for threshold $h$ can be set in order to achieve the maximum percentage of successful experiments increases when $\mu_{\text {after }}$ increases also. On the opposite, the MRL, the number of False alarms, the number of Change Fails, the number of $\hat{\tau}$ Fails and the number of successful experiments decreases when $\mu_{\text {after }}$ increases.

From the results presented in Table 4 it can be stated that the CUSUM algorithm for abrupt change detection can be successfully applied to data following a curcular normal distribution within the parameter values tested. 
Table 5 Mean Run Length (MRL) and mean change point estimate $\overline{\hat{\tau}}$ for same percentage of successful experiments in the case of an abrupt change. Note that both variables are approximately the same regardless the time detector $\delta$. The values were set to $\mu=0$ and $\sigma=25 \mathrm{~min}$. for all experiments. Each experiment with same parameters was performed 10,000 times. Light gray rows highlights experiments with the same pair of time detector $\delta$ and threshold $h$. There was no change fails in any experiment.

\begin{tabular}{cccccccc}
\hline$\beta$ & $\delta$ & $h$ & MRL & $\overline{\hat{\tau}}$ & $\begin{array}{c}\text { False } \\
\text { Alarms }\end{array}$ & $\begin{array}{c}\hat{\tau} \\
\text { Fails }\end{array}$ & Success \\
\hline 0.25 & 2.5 & 1.2 & $90.52 \pm 0.17$ & $65.17 \pm 0.10$ & 749 & 160 & 90.91 \\
0.25 & 5.0 & 2.0 & $90.43 \pm 0.17$ & $65.08 \pm 0.10$ & 919 & 173 & 89.08 \\
0.25 & 7.5 & 2.6 & $91.25 \pm 0.17$ & $65.26 \pm 0.10$ & 959 & 114 & 89.27 \\
0.25 & 10.0 & 3.2 & $93.95 \pm 0.17$ & $66.07 \pm 0.10$ & 798 & 118 & 90.84 \\
\hline 0.5 & 2.5 & 1.2 & $78.59 \pm 0.11$ & $55.65 \pm 0.16$ & 705 & 188 & 91.07 \\
0.5 & 5.0 & 2.0 & $78.11 \pm 0.11$ & $55.79 \pm 0.16$ & 917 & 154 & 89.29 \\
0.5 & 7.5 & 2.6 & $78.37 \pm 0.11$ & $55.92 \pm 0.16$ & 902 & 151 & 89.47 \\
0.5 & 10.0 & 3.2 & $79.73 \pm 0.11$ & $56.05 \pm 0.16$ & 795 & 130 & 90.75 \\
\hline 0.75 & 2.5 & 1.2 & $73.07 \pm 0.08$ & $51.38 \pm 0.14$ & 752 & 210 & 90.38 \\
0.75 & 5.0 & 2.0 & $72.63 \pm 0.08$ & $51.94 \pm 0.14$ & 871 & 155 & 89.74 \\
0.75 & 7.5 & 2.6 & $72.71 \pm 0.08$ & $51.93 \pm 0.14$ & 906 & 160 & 89.34 \\
0.75 & 10.0 & 3.2 & $73.58 \pm 0.08$ & $52.11 \pm 0.14$ & 763 & 127 & 91.10 \\
\hline 1.0 & 2.5 & 1.2 & $69.94 \pm 0.07$ & $49.67 \pm 0.13$ & 699 & 198 & 91.03 \\
1.0 & 5.0 & 2.0 & $69.44 \pm 0.07$ & $49.88 \pm 0.13$ & 890 & 180 & 89.30 \\
1.0 & 7.5 & 2.6 & $69.41 \pm 0.07$ & $50.18 \pm 0.13$ & 965 & 143 & 88.92 \\
1.0 & 10.0 & 3.2 & $69.90 \pm 0.07$ & $50.08 \pm 0.13$ & 785 & 152 & 90.63 \\
\hline 1.5 & 2.5 & 1.2 & $66.06 \pm 0.05$ & $47.37 \pm 0.12$ & 767 & 184 & 90.49 \\
1.5 & 5.0 & 2.0 & $65.61 \pm 0.05$ & $47.66 \pm 0.12$ & 899 & 175 & 89.26 \\
1.5 & 7.5 & 2.6 & $65.51 \pm 0.05$ & $47.90 \pm 0.12$ & 995 & 149 & 88.56 \\
1.5 & 10.0 & 3.2 & $65.71 \pm 0.05$ & $47.87 \pm 0.12$ & 800 & 147 & 90.53 \\
\hline 2.0 & 2.5 & 1.2 & $63.76 \pm 0.04$ & $46.48 \pm 0.11$ & 735 & 182 & 90.83 \\
2.0 & 5.0 & 2.0 & $63.31 \pm 0.04$ & $46.58 \pm 0.11$ & 882 & 172 & 89.46 \\
2.0 & 7.5 & 2.8 & $63.75 \pm 0.04$ & $46.50 \pm 0.11$ & 739 & 191 & 90.70 \\
2.0 & 10.0 & 3.2 & $63.39 \pm 0.04$ & $46.80 \pm 0.11$ & 776 & 168 & 90.56 \\
\hline & & & & & & &
\end{tabular}

4.2 Linear trend detection and change point estimation in a process following a circular normal distribution

The first step for detecting a change in a process following a circular normal distribution with a linear drift is to use the log-likelihood (Eq. 6), so a value for the detector time $\delta$ should be chosen. For doing that, we present first some experimental insights.

Fig. 7 shows the number of successful experiments with regards the threshold $h$ set, for the values $\delta \in\{2.5,5.0,7.5,10.0\}$ in minutes. Note that the same percentage of successful experiments were achieved for different values of the detector time $\delta$. So the first insight is that, once the detector time $\delta$ is set, the threshold $h$ can be chosen to approximately achieve the same percentage of successful experiments.

Table 5 shows the values for the MRL and the mean estimated change point $\overline{\hat{\tau}}$ for values of the linear drift rate $\beta \in\{0.25,0.5,0.75,1.0,1.5,2.0\}$ minutes per day. Once the detector time $\delta$ was set, the value for the threshold $h$ was chosen to approximately achieve the same percentage of successful experiments in all cases $(90 \%)$. Note that, for the same linear drift rate $\beta$, and once the values for time detector $\delta$ and threshold $h$ have been set to approximately achieve the same percentage of successful experiments, the differences in the MRL and the mean of the estimated change point $\overline{\hat{\tau}}$ are small. For example, in the case of 
Table $6 \mathrm{MRL}$ and mean change point estimate $\overline{\hat{\tau}}$ for different combinations of $\sigma$ in minutes, and drift rate $\beta$ in minutes per day, in the case of linear drift. The time of the detector was set to $\delta=2.5 \mathrm{~min}$. in all experiments. Threshold $h$ was set to approximately get $90 \%$ of successful experiments. Each experiment with same parameters was performed 10,000 times.

\begin{tabular}{|c|c|c|c|c|c|c|c|}
\hline$\sigma$ & $\beta$ & $h$ & $\begin{array}{l}\text { Mean Run } \\
\text { Length }\end{array}$ & $\overline{\bar{\tau}}$ & $\begin{array}{c}\text { False } \\
\text { Alarms }\end{array}$ & $\begin{array}{c}\hat{\tau} \\
\text { Fails }\end{array}$ & Success \\
\hline 40 & 0.25 & 0.8 & $99.80 \pm 0.21$ & $73.11 \pm 0.25$ & 670 & 188 & 90.68 \\
\hline 40 & 0.50 & 0.8 & $86.28 \pm 0.14$ & $61.45 \pm 0.19$ & 730 & 187 & 90.83 \\
\hline 40 & 0.75 & 0.8 & $79.54 \pm 0.11$ & $55.83 \pm 0.16$ & 668 & 223 & 91.09 \\
\hline 40 & 1.00 & 0.8 & $75.75 \pm 0.09$ & $53.28 \pm 0.15$ & 660 & 236 & 91.04 \\
\hline 40 & 1.50 & 0.8 & $70.82 \pm 0.07$ & $49.69 \pm 0.13$ & 648 & 234 & 91.18 \\
\hline 40 & 2.00 & 0.8 & $70.82 \pm 0.07$ & $49.69 \pm 0.13$ & 648 & 234 & 91.18 \\
\hline 30 & 0.25 & 1.0 & $93.33 \pm 0.18$ & $67.97 \pm 0.22$ & 829 & 186 & 89.83 \\
\hline 30 & 0.50 & 1.0 & $80.80 \pm 0.12$ & $57.83 \pm 0.17$ & 745 & 207 & 90.48 \\
\hline 30 & 0.75 & 1.0 & $80.80 \pm 0.12$ & $57.83 \pm 0.17$ & 745 & 207 & 90.48 \\
\hline 30 & 1.00 & 1.0 & $71.72 \pm 0.07$ & $50.99 \pm 0.13$ & 775 & 168 & 90.57 \\
\hline 30 & 1.50 & 1.0 & $71.72 \pm 0.07$ & $50.99 \pm 0.13$ & 775 & 168 & 90.57 \\
\hline 30 & 2.00 & 1.0 & $71.72 \pm 0.07$ & $50.99 \pm 0.13$ & 775 & 168 & 90.57 \\
\hline 25 & 0.25 & 1.2 & $90.27 \pm 0.17$ & $64.94 \pm 0.22$ & 720 & 200 & 90.80 \\
\hline 25 & 0.50 & 1.2 & $78.61 \pm 0.11$ & $55.56 \pm 0.17$ & 761 & 180 & 90.59 \\
\hline 25 & 0.75 & 1.2 & $73.16 \pm 0.08$ & $51.72 \pm 0.14$ & 731 & 185 & 90.84 \\
\hline 25 & 1.00 & 1.2 & $69.92 \pm 0.07$ & $49.66 \pm 0.13$ & 758 & 202 & 90.40 \\
\hline 25 & 1.50 & 1.2 & $66.19 \pm 0.05$ & $47.71 \pm 0.12$ & 751 & 191 & 90.58 \\
\hline 25 & 2.00 & 1.2 & $63.80 \pm 0.04$ & $46.42 \pm 0.11$ & 735 & 183 & 90.82 \\
\hline 20 & 0.25 & 1.4 & $85.64 \pm 0.15$ & $61.37 \pm 0.20$ & 874 & 181 & 89.45 \\
\hline 20 & 0.50 & 1.4 & $74.99 \pm 0.09$ & $53.43 \pm 0.15$ & 831 & 181 & 89.88 \\
\hline 20 & 0.75 & 1.4 & $70.33 \pm 0.07$ & $50.40 \pm 0.13$ & 823 & 194 & 89.83 \\
\hline 20 & 1.00 & 1.4 & $67.45 \pm 0.06$ & $48.44 \pm 0.13$ & 836 & 194 & 89.70 \\
\hline 20 & 1.50 & 1.4 & $64.04 \pm 0.05$ & $46.88 \pm 0.11$ & 859 & 178 & 89.63 \\
\hline 20 & 2.00 & 1.4 & $62.01 \pm 0.04$ & $46.09 \pm 0.11$ & 822 & 165 & 90.13 \\
\hline 15 & 0.25 & 1.8 & $81.38 \pm 0.12$ & $57.60 \pm 0.17$ & 762 & 184 & 90.54 \\
\hline 15 & 0.50 & 1.8 & $71.93 \pm 0.07$ & $50.95 \pm 0.14$ & 778 & 195 & 90.27 \\
\hline 15 & 0.75 & 1.8 & $67.64 \pm 0.06$ & $48.35 \pm 0.13$ & 745 & 181 & 90.74 \\
\hline 15 & 1.00 & 1.8 & $65.09 \pm 0.05$ & $47.33 \pm 0.12$ & 754 & 183 & 90.63 \\
\hline 15 & 1.50 & 1.8 & $62.06 \pm 0.04$ & $45.89 \pm 0.11$ & 783 & 158 & 90.59 \\
\hline 15 & 2.00 & 1.8 & $60.30 \pm 0.03$ & $45.55 \pm 0.10$ & 802 & 140 & 90.58 \\
\hline 10 & 0.25 & 2.4 & $75.84 \pm 0.09$ & $53.61 \pm 0.15$ & 783 & 146 & 90.71 \\
\hline 10 & 0.50 & 2.4 & $67.68 \pm 0.06$ & $48.56 \pm 0.13$ & 817 & 175 & 90.08 \\
\hline 10 & 0.75 & 2.4 & $64.18 \pm 0.04$ & $46.73 \pm 0.12$ & 792 & 182 & 90.26 \\
\hline 10 & 1.00 & 2.4 & $62.00 \pm 0.04$ & $46.02 \pm 0.11$ & 775 & 152 & 90.73 \\
\hline 10 & 1.50 & 2.4 & $59.51 \pm 0.03$ & $45.36 \pm 0.10$ & 756 & 131 & 91.13 \\
\hline 10 & 2.00 & 2.4 & $58.04 \pm 0.02$ & $45.19 \pm 0.09$ & 805 & 126 & 90.69 \\
\hline
\end{tabular}

$\beta=0.25$ minutes per day, the maximum of the MRL is $93.95 \pm 0.17(\delta=10, h=$ $3.2)$, and the minimum of the MRL is $90.43 \pm 0.17(\delta=5.0, h=2.0)$, which difference is $3.53 \pm 0.34(3.89 \%)$. For the estimated change point $\hat{\tau}$ the maximum is $66.07 \pm 0.10(\delta=10.0, h=3.2)$, the minimum is $65.08 \pm 0.10(\delta=5.0, h=2.0)$, which difference is $0.99 \pm 0.20(1.52 \%)$. In the case of $\beta=2.0$ minutes per day, the maximum of the MRL is $63.76 \pm 0.04(\delta=2.5, h=1.2)$, and the minimum of the MRL is $63.31 \pm 0.04(\delta=5.0, h=2.0)$, which difference is $0.45 \pm 0.08(0.71 \%)$. For the estimated change point $\hat{\tau}$ the maximum is $46.80 \pm 0.11(\delta=10.0, h=3.2)$, the minimum is $46.48 \pm 0.11(\delta=2.5, h=1.2)$, which difference is $0.32 \pm 0.22(0.69 \%)$. There was no change fails in any experiment. This is the second insight, if the values for $\delta$ and $h$ are set to achieve some percentage of successful experiments, 


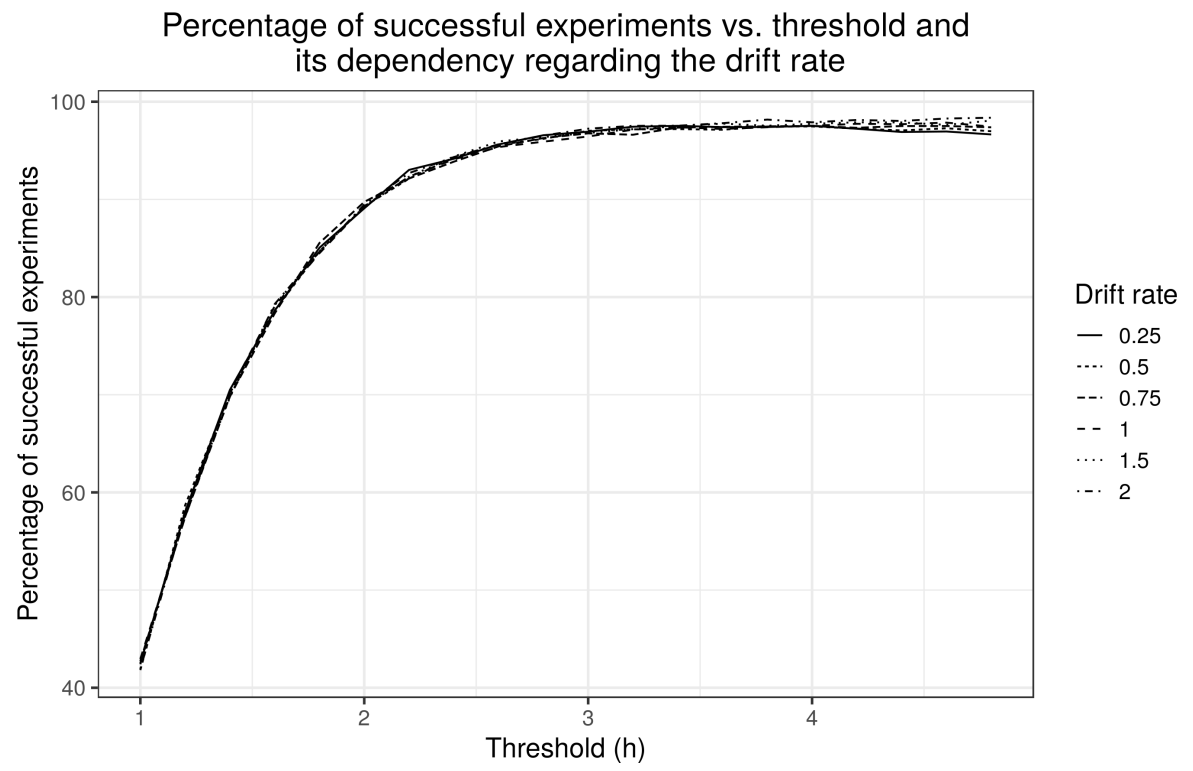

Fig. 8 Percentage of successful experiments vs. threshold for different drift rates in minutes per day. $\sigma=25 \mathrm{~min}$. and $t_{\text {detector }}=5.0 \mathrm{~min}$. for all experiments.

the MRL and the mean of the estimated change point $\overline{\hat{\tau}}$ are approximately the same for any linear drift rate $\beta$.

As a conclusion, and taken the two previous insights, it can be stated that for any given time detector $\delta$, the threshold $h$ can be set to achieve a percentage of successful experiments, which in turn gives the same result for the MRL and the mean estimated change point $\overline{\hat{\tau}}$.

A third important insight can be extracted from Table 5 if one looks at the light grey highlighted rows: once the time detector $\delta$ is set, and independently of the linear drift rate $\beta$, the threshold $h$ can be chosen to obtain the same percentage of successful experiments. Fig. 8 shows this behavior in detail: for the six values of $\beta \in\{0.25,0.50,0.75,1.00,1.50,2.00\}$ minutes per day, the percentage of successful experiments as a function of the threshold $h$ are quite similar (the profile of the six plots behave the same). Therefore, it can be concluded that once the detector time $\delta$ is set, the threshold $h$ can be chosen to achieve the same percentage of successful experiments regardless the linear drift rate $\beta$ of the process. This conclusion is important because it allows us to compare the MRL and mean estimated change point $\overline{\hat{\tau}}$ for different linear drift rates $\beta$ just setting the threshold $h$, which in turn sets the percentage of successful experiments.

Table 6 shows the MRL and the mean estimated change point $\overline{\hat{\tau}}$ for different values of $\sigma$ in minutes, and linear drift rate $\beta$ in minutes per day. For all experiments the change point was set to $\tau=50$, the detector time was set to $\delta=2.5$ minutes, and the threshold $h$ was chosen to approximately achieve $90 \%$ of successful experiments. Note that the value for the chosen threshold was the same for all linear drift rates $\beta$ for each value of $\sigma$, as it was concluded in the former paragraph. 
The biggest MRL in Table 6 is $99.80 \pm 0.21$, which corresponds to a value for $\sigma=40$ minutes, and linear drift rate $\beta=0.25$ minutes per day. In this case, the change was detected 49.80 days after it happened. After these 49.80 days, the mean of the circular normal process is $\mu=49.80 \times 0.25=12.45$ minutes. This could seem a big delay for detecting an anomalous behavior, but it is not so big when compared with the value of $\sigma=40 \mathrm{~min}$. in this case, in fact, this is the most challenging experiment in the series of performed experiments. Also, all processes were random, and the drift rate was linear, so the difference between the original mean and the final mean increments 0.25 minutes per day only. In the case of $\sigma=10 \mathrm{~min}$., and $\beta=0.25$ minutes per day, the MRL was $75.84 \pm 0.09$, so the change in the process was detected 25.84 days after it happened, and the mean of the process at this time was $\mu=6.25$ minutes. Note the same behavior for other values of $\sigma$ and $\beta$ from Table 6 , the smaller the value for $\sigma$ the quicker the change was detected. Also, for the same value of $\sigma$, when the linear drift increases the MRL decreases.

The biggest difference between the real value of the change point $\tau(50)$ and the mean estimated change point $\overline{\hat{\tau}}$ is $23.11 \mathrm{pm} 0.25$ days, corresponds to $\sigma=40 \mathrm{~min}$. and $\beta=0.25$ minutes per day, which is the most challenging case tested: there is a high variability in the data $\sigma$ and a low drift rate $\beta$. The second biggest difference is 17.97 which corresponds to $\sigma=30 \mathrm{~min}$. and $\beta=0.25$ minutes per day. In the other cases, the differences ranges between $0.40 \leq\|\tau-\overline{\hat{\tau}}\| \leq 14.94$ days. The average difference between the real value of the change point and the mean estimated change point is $4.90_{+3.17}^{-1.98}$ days for all set of experiments performed.

The results from the experiments show that the proposed method can be used to first detect, and then estimate a linear change in data series following a circular normal distribution.

\section{Conclusions}

A new method to detect linear behavior drift in processes following a circular normal distribution is presented in this work. This method is based on the used of multiple hypothesis to estimate the linear drift rate of a process following a circular normal distribution. The method was applied on public ADLs data sets which were modeled as mixtures of circular normal distribution. The method is able at detecting first, and then estimate, a change point in a time series. The circular normal distribution is used to model periodic data, which is the case of Activities of Daily Living. Experimental results have shown the validity of the method in detecting and estimating the change point in time.

Some examples of application of the proposed method are: to detect, and estimate, the start of a cognitive or physical decay of a monitorized older adult; or to detect, and estimate, the change in ADL due to a replacement in the medication a person takes. These may be of great interest in the health realm, as it can help health practitioners to better understand behavioural changes in patients.

A key point in the method is to select a threshold $h$ to detect the change, the lower the threshold, the more false positives detected. Taken this insight, a manifold strategy can be developed to supervise the behavior of a monitorized person. First, a low threshold can be used to check for change in ADL, if the threshold was reached, a first level of alarm can be set, and a particular supervision 
of the person could be established; then the threshold was increased, and if the new threshold was reached a higher level alarm could be set and special supervision, or even some kind of intervention, could be placed.

Although the MRL could seem quite long in the tested challenging case (41 days for $\beta=0.25$ and $\sigma=40 \mathrm{~min}$.), this delay is not so long when compared with the time between reported onset of symptoms and diagnosis, which is reported as 2.8 years [96].

In order to asses the validity of the algorithm using real data, we have planned to perform a set of experiments with volunteers. The data will be get, in part, with an improved version of the acquisition system developed by the members of our research group [97].

Funding information This work has been partially funded by the Spanish Ministry of Science, Innovation and Universities through the "Retos investigación" programme (RTI2018-095168-B-C53) and by Jaume I University "Pla de promoció de la investigació 2017" programme (UJI-B2017-45). Óscar Belmonte-Fernández had a grant by the Spanish Ministry of Science, Innovation and Universities (PRX18/00123) for developing part of this work.

\section{Compliance with Ethical Standards}

Conflict of Interest The authors declare that they have no conflict of interest. Ethical Approval This article does not contain any studies with human participants or animals performed by any of the authors.

\section{References}

1. Austin J, Dodge HH, Riley T, Jacobs PG, Thielke S, Kaye J. A Smart-Home System to Unobtrusively and Continuously Assess Loneliness in Older Adults. IEEE Journal of Translational Engineering in Health and Medicine. 2016;4:1-11.

2. Fozard JL, Rietsema J, Bouma H, Graafmans JAM. Gerontechnology: Creating enabling environments for the challenges and opportunities of aging. Educational Gerontology. $2000 ; 26(4): 331-344$.

3. Rashidi P, Mihailidis A. A Survey on Ambient-Assisted Living Tools for Older Adults. IEEE Journal of Biomedical and Health Informatics. 2013 May;17(3):579-590.

4. Bjering H, Curry J, Maeder A. Gerontechnology: the importance of user participation in ICT development for older adults. In: Investing in E-Health: People, Knowledge and Technology for a Healthy Future: Selected Papers from the 22nd Australian National Health Informatics Conference (HIC 2014), 11-14 August 2014, Melbourne Convention and Exhibition Centre; 2014. p. 7-12.

5. Banaee H, Ahmed MU, Loutfi A. Data Mining for Wearable Sensors in Health Monitoring Systems: A Review of Recent Trends and Challenges. Sensors. 2013;13(12):17472-17500.

6. Kim JY, Liu N, Tan HX, Chu CH. Unobtrusive Monitoring to Detect Depression for Elderly With Chronic Illnesses. IEEE Sensors Journal. 2017;17(17):5694-5704.

7. Igual R, Medrano C, Plaza I. Challenges, issues and trends in fall detection systems. Biomedical engineering online. 2013;12(1):66.

8. Chandola V, Banerjee A, Kumar V. Anomaly Detection: A Survey. ACM Comput Surv. 2009 Jul;41(3):15:1-15:58. 
9. Lee W, Stolfo SJ, et al. Data Mining Approaches for Intrusion Detection. In: USENIX Security Symposium. San Antonio, TX; 1998. p. 79-93.

10. Atli BG, Miche Y, Kalliola A, Oliver I, Holtmanns S, Lendasse A. Anomaly-Based Intrusion Detection Using Extreme Learning Machine and Aggregation of Network Traffic Statistics in Probability Space. Cognitive Computation. 2018 Jun;.

11. Stoumbos ZG, Reynolds Jr MR, Ryan TP, Woodall WH. The state of statistical process control as we proceed into the 21st century. Journal of the American Statistical Association. 2000;95(451):992-998.

12. Tsui FC, Espino JU, Dato VM, Gesteland PH, Hutman J, Wagner MM. Technical description of RODS: a real-time public health surveillance system. Journal of the American Medical Informatics Association. 2003;10(5):399-408.

13. Barnett TP, Pierce DW, Schnur R. Detection of anthropogenic climate change in the world's oceans. Science. 2001;292(5515):270-274.

14. Oakland JS. Statistical process control. Routledge; 2007.

15. Page ES. Continuous Inspection Schemes. Biometrika. 1954;41(1/2):pp. 100-115.

16. Page ES. A Test for a Change in a Parameter Occurring at an Unknown Point. Biometrika. 1955;42(3/4):pp. 523-527.

17. MacDonald SW, Hultsch DF, Dixon RA. Performance variability is related to change in cognition: Evidence from the Victoria Longitudinal Study. Psychology and aging. 2003;18(3):510.

18. Jimison H, Pavel M, McKanna J, Pavel J. Unobtrusive monitoring of computer interactions to detect cognitive status in elders. IEEE Transactions on Information Technology in Biomedicine. 2004 Sept;8(3):248-252.

19. Hayes TL, Abendroth F, Adami A, Pavel M, Zitzelberger TA, Kaye JA. Unobtrusive assessment of activity patterns associated with mild cognitive impairment. Alzheimer's \& dementia: the journal of the Alzheimer's Association. 2008;4(6):395-405.

20. Dodge HH, Mattek NC, Austin D, Hayes TL, Kaye JA. In-home walking speeds and variability trajectories associated with mild cognitive impairment. Neurology. 2012;78(24):1946-1952.

21. Boise L, Camicioli R, Morgan DL, Rose JH, Congleton L. Diagnosing dementia: perspectives of primary care physicians. The Gerontologist. 1999;39(4):457-464.

22. Organization WH. The World Health Report 2001: Mental health: new understanding, new hope. World Health Organization; 2001.

23. Bradford A, Kunik ME, Schulz P, Williams SP, Singh H. Missed and delayed diagnosis of dementia in primary care: prevalence and contributing factors. Alzheimer disease and associated disorders. 2009;23(4):306.

24. Gaugler J, James B, Johnson T, Scholz K, Weuve J. 2016 Alzheimer's disease facts and figures. Alzheimer's and Dementia. 2016;12(4):459-509.

25. Organization WH. World report on ageing and health. World Health Organization; 2015.

26. Pires P, Mendes L, Mendes J, Rodrigues R, Pereira A. Integrated e-Healthcare System for Elderly Support. Cognitive Computation. 2016 Apr;8(2):368-384.

27. Castillo JC, Castro-González Á, Fernández-Caballero A, Latorre JM, Pastor JM, Fernández-Sotos A, et al. Software Architecture for Smart Emotion Recognition and Regulation of the Ageing Adult. Cognitive Computation. 2016 Apr;8(2):357-367.

28. Alberdi Aramendi A, Weakley A, Aztiria Goenaga A, Schmitter-Edgecombe M, Cook DJ. Automatic assessment of functional health decline in older adults based on smart home data. Journal of Biomedical Informatics. 2018;81:119-130.

29. Galambos C, Skubic M, Wang S, Rantz M. Management of dementia and depression utilizing in-home passive sensor data. Gerontechnology: international journal on the fundamental aspects of technology to serve the ageing society. 2013;11(3):457.

30. Jessen F, Amariglio RE, van Boxtel M, Breteler M, Ceccaldi M, Chételat G, et al. A conceptual framework for research on subjective cognitive decline in preclinical Alzheimer's disease. Alzheimer's and Dementia. 2014;10(6):844 - 852 .

31. Guarnieri B, Sorbi S. Sleep and Cognitive Decline: A Strong Bidirectional Relationship. It Is Time for Specific Recommendations on Routine Assessment and the Management of Sleep Disorders in Patients with Mild Cognitive Impairment and Dementia. European Neurology. 2015;74(1-2):43-48.

32. Hall CB, Lipton RB, Sliwinski M, Stewart WF. A change point model for estimating the onset of cognitive decline in preclinical Alzheimer's disease. Statistics in Medicine. 2000 jun;19(11-12):1555-1566. 
33. van Someren EJW, Hagebeuk EEO, Lijzenga C, Scheltens P, de Rooij SEJA, Jonker C, et al. Circadian rest-activity rhythm disturbances in alzheimer's disease. Biological Psychiatry. 1996 aug;40(4):259-270.

34. Schmidt C, Peigneux P, Cajochen C. Age-Related Changes in Sleep and Circadian Rhythms: Impact on Cognitive Performance and Underlying Neuroanatomical Networks. Frontiers in Neurology. 2012 jul;3:118.

35. Leng Y, Musiek ES, Hu K, Cappuccio FP, Yaffe K. Association between circadian rhythms and neurodegenerative diseases. The Lancet Neurology. 2019;18(3):307-318.

36. Kaye JA, Maxwell SA, Mattek N, Hayes TL, Dodge H, Pavel M, et al. Intelligent Systems For Assessing Aging Changes: home-based, unobtrusive, and continuous assessment of aging. The journals of gerontology Series B, Psychological sciences and social sciences. 2011 jul;66 Suppl 1(Suppl 1):i180-90.

37. Keage HAD, Banks S, Yang KL, Morgan K, Brayne C, Matthews FE. What sleep characteristics predict cognitive decline in the elderly? Sleep Medicine. 2012 aug;13(7):886-892.

38. Lyons BE, Austin D, Seelye A, Petersen J, Yeargers J, Riley T, et al. Pervasive computing technologies to continuously assess Alzheimer's disease progression and intervention efficacy. Frontiers in Aging Neuroscience. 2015 jun;7(JUN):102.

39. Peter-Derex L, Yammine P, Bastuji H, Croisile B. Sleep and Alzheimer's disease. W.B. Saunders; 2015.

40. Seelye A, Mattek N, Howieson D, Riley T, Wild K, Kaye J. The impact of sleep on neuropsychological performance in cognitively intact older adults using a novel in-home sensor-based sleep assessment approach. The Clinical neuropsychologist. 2015;29(1):53-66.

41. Devore EE, Grodstein F, Schernhammer ES. Sleep Duration in Relation to Cognitive Function among Older Adults: A Systematic Review of Observational Studies. Neuroepidemiology. 2016;46(1):57-78.

42. Cassidy-Eagle EL, Siebern A. Sleep and mild cognitive impairment. Sleep Science and Practice. 2017 dec;1(1):15.

43. Liguori C, Romigi A, Nuccetelli M, Zannino S, Sancesario G, Martorana A, et al. Orexinergic system dysregulation, sleep impairment, and cognitive decline in Alzheimer disease. JAMA neurology. 2014;71(12):1498-1505.

44. Mohs RC, Schmeidler J, Aryan M. Longitudinal studies of cognitive, functional and behavioural change in patients with Alzheimer's disease. Statistics in Medicine. 2000 jun;19(11-12):1401-1409.

45. Stem RG, Mohs RC, D P, Davidson M, Silverman J, Kramer-ginsberg E, et al. A Longitudinal Study of Alzheimer's Disease. Measurement, Rate, and Predictors of Cognitive Deterioration. Psychiatry: Interpersonal and Biological Processes. 1994;(March):390-396.

46. Petersen J, Austin D, Mattek N, Kaye J. Time out-of-home and cognitive, physical, and emotional wellbeing of older adults: A longitudinal mixed effects model. PLoS ONE. 2015 oct;10(10):e0139643.

47. Harada K, Lee S, Lee S, Bae S, Harada K, Suzuki T, et al. Objectively-measured outdoor time and physical and psychological function among older adults. Geriatrics \& Gerontology International. 2016 oct;17(10):1455-1462.

48. Kötteritzsch A, Weyers B. Assistive Technologies for Older Adults in Urban Areas: A Literature Review. Cognitive Computation. 2016 Apr;8(2):299-317.

49. Zarandi MHF, Alaeddini A. A general fuzzy-statistical clustering approach for estimating the time of change in variable sampling control charts. Information Sciences. 2010;180(16):3033 - 3044.

50. López-de Ipiña K, Alonso JB, Solé-Casals J, Barroso N, Henriquez P, Faundez-Zanuy M, et al. On Automatic Diagnosis of Alzheimer's Disease Based on Spontaneous Speech Analysis and Emotional Temperature. Cognitive Computation. 2015 Feb;7(1):44-55.

51. Impedovo D, Pirlo G, Vessio G, Angelillo MT. A Handwriting-Based Protocol for Assessing Neurodegenerative Dementia. Cognitive Computation. 2019 May;.

52. Shewhart WA. Economic control of quality of manufactured product. vol. 509. ASQ Quality Press; 1931.

53. Roberts S. Control chart tests based on geometric moving averages. Technometrics. 1959;1(3):239-250.

54. Basseville M, Nikiforov IV. Detection of Abrupt Changes: Theory and Application. Upper Saddle River, NJ, USA: Prentice-Hall, Inc.; 1993.

55. Cheng CS. A multi-layer neural network model for detecting changes in the process mean. vol. 28 ; 1995. p. $51-61$. 
56. Guh RS, Hsieh YC. A neural network based model for abnormal pattern recognition of control charts. Computers \& Industrial Engineering. 1999;36(1):97 - 108.

57. Cheng CS, Cheng SS. A neural network-based procedure for the monitoring of exponential mean. Computers \& Industrial Engineering. 2001;40(4):309 - 321.

58. Chang S, Aw C. A neural fuzzy control chart for detecting and classifying process mean shifts. International Journal of production research. 1996;34(8):2265-2278.

59. El-Shal SM, Morris AS. A fuzzy expert system for fault detection in statistical process control of industrial processes. Systems, Man, and Cybernetics, Part C: Applications and Reviews, IEEE Transactions on. 2000;30(2):281-289.

60. Alaeddini A, Ghazanfari M, Nayeri MA. A hybrid fuzzy-statistical clustering approach for estimating the time of changes in fixed and variable sampling control charts. Information sciences. 2009;179(11):1769-1784.

61. Lu KP, Chang ST, Yang MS. Change-point detection for shifts in control charts using fuzzy shift change-point algorithms. Computers \& Industrial Engineering. 2016;93:12-27.

62. Raftery A, Akman V. Bayesian analysis of a Poisson process with a change-point. Biometrika. 1986;p. 85-89.

63. Assareh H, Noorossana R, Mengersen KL. Bayesian change point estimation in Poissonbased control charts. Journal of Industrial Engineering International. 2013;9(1):32.

64. Perry MB, Pignatiello JJ, Simpson JR. Estimating the change point of a Poisson rate parameter with a linear trend disturbance. Quality and Reliability Engineering International. $2006 ; 22(4): 371-384$

65. Noorossana R, Shadman A. Estimating the change point of a normal process mean with a monotonic change. Quality and Reliability Engineering International. 2009;25(1):79-90.

66. Noorossana R, Heydari M. Change point estimation of a process variance with a linear trend disturbance. J Ind Eng Int. 2009;2:25-30.

67. Lara OD, Labrador MA. A Survey on Human Activity Recognition using Wearable Sensors. IEEE Communications Surveys Tutorials. 2013 Third;15(3):1192-1209.

68. Guan D, Ma T, Yuan W, Lee YK, Jehad Sarkar A. Review of sensor-based activity recognition systems. IETE Technical Review. 2011;28(5):418-433.

69. Ke SR, Thuc HLU, Lee YJ, Hwang JN, Yoo JH, Choi KH. A review on video-based human activity recognition. Computers. 2013;2(2):88-131.

70. Vrigkas M, Nikou C, Kakadiaris IA. A review of human activity recognition methods. Frontiers in Robotics and AI. 2015;2:28

71. van Kasteren TL, Englebienne G, Kröse BJ. Human activity recognition from wireless sensor network data: Benchmark and software. In: Activity recognition in pervasive intelligent environments. Springer; 2011. p. 165-186.

72. Mardia KV, Jupp PE. Directional statistics. vol. 494. John Wiley \& Sons; 2009.

73. Diethe T, Twomey N, Flach P. Bayesian modelling of the temporal aspects of smart home activity with circular statistics. In: Joint European Conference on Machine Learning and Knowledge Discovery in Databases. Springer; 2015. p. 279-294.

74. Chinellato E, Mardia K, Hogg D, Cohn A. An Incremental von Mises Mixture Framework for Modelling Human Activity Streaming Data. In: International Work-Conference on Time Series Analysis (ITISE 2017);. p. 379-389.

75. Brunsdon C, Corcoran J. Using circular statistics to analyse time patterns in crime incidence. Computers, Environment and Urban Systems. 2006;30(3):300 - 319.

76. Kubiak T, Jonas C. Applying circular statistics to the analysis of monitoring data. European Journal of Psychological Assessment. 2007;23(4):227-237.

77. Franco C, Demongeot J, Villemazet C, Vuillerme N. Behavioral telemonitoring of the elderly at home: Detection of nycthemeral rhythms drifts from location data. In: 2010 IEEE 24th International Conference on Advanced Information Networking and Applications Workshops. IEEE; 2010. p. 759-766.

78. Jammalamadaka SR, Sengupta A. Topics in circular statistics. vol. 5. World Scientific; 2001.

79. Yin X, Shen W, Samarabandu J, Wang X. Human activity detection based on multiple smart phone sensors and machine learning algorithms. In: 2015 IEEE 19th International Conference on Computer Supported Cooperative Work in Design (CSCWD); 2015. p. $582-587$.

80. Yin J, Yang Q, Pan JJ. Sensor-Based Abnormal Human-Activity Detection. IEEE Transactions on Knowledge and Data Engineering. 2008 Aug;20(8):1082-1090.

81. van Kasteren. Datasets for Activity Recognition; accessed 10/26/2017. Available from: https://sites.google.com/site/tim0306/datasets. 
82. Forkan ARM, Khalil I, Tari Z, Foufou S, Bouras A. A context-aware approach for longterm behavioural change detection and abnormality prediction in ambient assisted living. Pattern Recognition. 2015;48(3):628 - 641.

83. Zhu C, Sheng W, Liu M. Wearable Sensor-Based Behavioral Anomaly Detection in Smart Assisted Living Systems. IEEE Transactions on Automation Science and Engineering. 2015;12:1225-1234.

84. Duong TV, Bui HH, Phung DQ, Venkatesh S. Activity Recognition and Abnormality Detection with the Switching Hidden Semi-Markov Model. In: Proceedings of the 2005 IEEE Computer Society Conference on Computer Vision and Pattern Recognition (CVPR'05) Volume 1 - Volume 01. CVPR '05. Washington, DC, USA: IEEE Computer Society; 2005. p. $838-845$.

85. Shin JH, Lee B, Park KS. Detection of Abnormal Living Patterns for Elderly Living Alone Using Support Vector Data Description. IEEE Transactions on Information Technology in Biomedicine. 2011 May;15(3):438-448.

86. Palaniappan A, Bhargavi R, Vaidehi V. Abnormal human activity recognition using SVM based approach. In: 2012 International Conference on Recent Trends in Information Technology; 2012. p. 97-102.

87. Riboni D, Bettini C, Civitarese G, Janjua ZH, Helaoui R. SmartFABER: Recognizing finegrained abnormal behaviors for early detection of mild cognitive impairment. Artificial Intelligence in Medicine. 2016;67(Supplement C):57 - 74.

88. Virone G, Noury N, Demongeot J. A system for automatic measurement of circadian activity deviations in telemedicine. IEEE Transactions on Biomedical Engineering. 2002;49(12):1463-1469.

89. Fahad LG, Rajarajan M. Anomalies detection in smart-home activities. In: Machine Learning and Applications (ICMLA), 2015 IEEE 14th International Conference on. IEEE; 2015. p. 419-422.

90. Fisher NI. Statistical analysis of circular data. Cambridge University Press; 1995.

91. Cook DJ, Schmitter-Edgecombe M. Assessing the quality of activities in a smart environment. Methods of information in medicine. 2009;48(05):480-485.

92. Cook DJ. Learning setting-generalized activity models for smart spaces. IEEE intelligent systems. 2010;2010(99):1.

93. Hornik K, Grün B. movMF: an R package for fitting mixtures of von Mises-Fisher distributions. Journal of Statistical Software. 2014;58(10):1-31.

94. Kullback S, Leibler RA. On Information and Sufficiency. The Annals of Mathematical Statistics. 1951;22(1):79-86.

95. Dawadi PN, Cook DJ, Schmitter-Edgecombe M, Parsey C. Automated assessment of cognitive health using smart home technologies. Technology and Health Care. 2013 jan;21(4):323-343.

96. Brookmeyer R, Corrada MM, Curriero FC, Kawas C. Survival following a diagnosis of Alzheimer disease. Archives of neurology. 2002;59(11):1764-1767.

97. Belmonte-Fernández Ó, Puertas-Cabedo A, Torres-Sospedra J, Montoliu-Colás R, TrillesOliver S. An indoor positioning system based on wearables for ambient-assisted living. Sensors. 2017;17(1):36. 